\title{
Asymptotic expansions and approximations for the Caputo derivative
}

\author{
Yuri Dimitrov • Radan Miryanov • \\ Venelin Todorov
}

Received: date / Accepted: date

\begin{abstract}
In this paper we use the asymptotic expansions of the binomial coefficients and the weights of the L1 approximation to obtain approximations of order $2-\alpha$ and second-order approximations of the Caputo derivative by modifying the weights of the shifted Grünwald-Letnikov difference approximation and the L1 approximation of the Caputo derivative. A modification of the shifted Grünwald-Letnikov approximation is obtained which allows secondorder numerical solutions of fractional differential equations with arbitrary values of the solutions and their first derivatives at the initial point.
\end{abstract}

Keywords Binomial coefficient · Asymptotic expansion · Approximation of the Caputo derivative · Numerical solution

Mathematics Subject Classification (2000) 11B65 - 34A07 · 34E05 . 65 D30

The third author is supported by the Bulgarian Academy of Sciences through the Program for Career Development of Young Scientists, Grant DFNP-17-88/28.07.2017, Project Efficient Numerical Methods with an Improved Rate of Convergence for Applied Computational Problems, by the Bulgarian National Fund of Science under Project DN 12/5-2017, Project Efficient Stochastic Methods and Algorithms for Large-Scale Problems, and Project DN 12/4-2017, Project Advanced Analytical and Numerical Methods for Nonlinear Differential Equations with Applications in Finance and Environmental Pollution.

Y. Dimitrov

Department of Mathematics and Physics, University of Forestry, Sofia 1756, Bulgaria

E-mail: yuri.dimitrov@ltu.bg

R. Miryanov

Department of Statistics and Applied Mathematics, University of Economics, Varna 9002, Bulgaria E-mail: miryanov@ue-varna.bg

V. Todorov

Institute of Mathematics and Informatics, Bulgarian Academy of Sciences,

Acad. G. Bonchev Str, bl. 8, Sofia 1113, Bulgaria E-mail: vtodorov@math.bas.bg

Institute of Information and Communication Technologies, Bulgarian Academy of Sciences, Acad. G. Bonchev Str, bl. 25A, Sofia 1113, Bulgaria E-mail: venelin@parallel.bas.bg 


\section{Introduction}

The Caputo and Riemann-Liouville fractional derivatives are the two main approaches for generalizing the integer order derivatives. When $0<\alpha<1$ the Caputo and Riemann-Liouville derivatives with a lower limit at the point zero are defined as

$$
\begin{gathered}
y^{(\alpha)}(x)=D^{\alpha} y(x)=\frac{1}{\Gamma(1-\alpha)} \int_{0}^{x} \frac{y^{\prime}(t)}{(x-t)^{\alpha}} d t \\
D_{R L}^{\alpha} y(x)=\frac{1}{\Gamma(1-\alpha)} \frac{d}{d x} \int_{0}^{x} \frac{y(t)}{(x-t)^{\alpha}} d t .
\end{gathered}
$$

The Caputo and Riemann-Liouville derivatives are related as

$$
D_{R L}^{\alpha} y(x)=D^{\alpha} y(x)+\frac{y(0)}{\Gamma(1-\alpha) x^{\alpha}} .
$$

The Caputo derivative is a suitable choice for a fractional derivative in fractional differential equations. Fractional differential equations is a growing field of mathematics with applications in finance, bioengineering, control theory, quantum mechanics (Magin 2004; Wang ang Xu 2007; Monje et al. 2010; Zhang et al. 2016). The finite difference schemes for numerical solution of fractional differential equations involve approximations for the fractional derivative. Let $h=x / N$ and $y_{\beta}=y(\beta h)$ for $0 \leq \beta \leq N$. The Grünwald-Letnikov difference approximation is a first-order approximation of the Riemann-Liouville derivative, when $y \in C^{1}[0, x]$ and it is a first-order approximation of the Caputo derivative when the function $y$ satisfies the condition $y(0)=0$ :

$$
A_{N}^{G L}[y(x)]=\frac{1}{h^{\alpha}} \sum_{k=0}^{N-1}(-1)^{k}\left(\begin{array}{l}
\alpha \\
k
\end{array}\right) y(x-k h)=y^{(\alpha)}(x)+O(h) .
$$

When the function $y \in C^{2}[0, x]$ and satisfies the condition $y(0)=y^{\prime}(0)=0$, the Grünwald-Letnikov approximation is a second-order approximation for the Caputo derivative at the point $x-\alpha h / 2$ :

$$
\frac{1}{h^{\alpha}} \sum_{k=0}^{N-1}(-1)^{k}\left(\begin{array}{l}
\alpha \\
k
\end{array}\right) y(x-k h)=y^{(\alpha)}(x-\alpha h / 2)+O\left(h^{2}\right) .
$$

The weights $w_{k}^{(\alpha)}=(-1)^{k}\left(\begin{array}{l}\alpha \\ k\end{array}\right)$ of the Grünwald-Letnikov approximation involve the binomial coefficients defined as:

$$
\left(\begin{array}{l}
\alpha \\
k
\end{array}\right)=\frac{\Gamma(\alpha+1)}{\Gamma(k+1) \Gamma(\alpha-k+1)}=\frac{\alpha(\alpha-1) \cdots(\alpha-k+1)}{k !} .
$$

The binomial coefficients satisfy the identities

$$
(-1)^{k}\left(\begin{array}{l}
\alpha \\
k
\end{array}\right)=\left(\begin{array}{c}
k-\alpha-1 \\
k
\end{array}\right)=\frac{\Gamma(k-\alpha)}{\Gamma(k+1) \Gamma(-\alpha)},\left(\begin{array}{l}
\alpha-1 \\
k-1
\end{array}\right)+\left(\begin{array}{c}
\alpha-1 \\
k
\end{array}\right)=\left(\begin{array}{l}
\alpha \\
k
\end{array}\right),
$$


and the gamma function satisfies the asymptotic formula (Podlubny 1999)

$$
k^{b-a} \frac{\Gamma(k+a)}{\Gamma(k+b)}=1+O\left(k^{-1}\right) .
$$

The weights of the Grünwald-Letnikov approximation are the coefficients of the binomial series of the function $(1-x)^{\alpha}$ and the coefficients of the right endpoint asymptotic expansion of the Grünwald-Letnikov approximation are equal to the coefficients of the series expansion of the function $\left(1-e^{-x}\right)^{\alpha} / x^{\alpha}$ at the point $x=0$ :

$$
\left(\frac{1-e^{-x}}{x}\right)^{\alpha}=\sum_{k=0}^{\infty} \frac{B_{k}^{(-\alpha)}(-\alpha)}{k !} x^{k}
$$

where $B_{k}^{(-\alpha)}(x)$ are the generalized Bernoulli polynomials. When the function $y \in C^{m}[0, x]$ and satisfies the condition $y^{(k)}(0)=0$, for $k=0,1, \ldots, m$, the Grünwald-Letnikov approximation has an asymptotic expansion of order $m$ :

$\frac{1}{h^{\alpha}} \sum_{k=0}^{N-1}(-1)^{k}\left(\begin{array}{l}\alpha \\ k\end{array}\right) y(x-k h)=y^{(\alpha)}(x)+\sum_{k=1}^{m-1} \frac{B_{k}^{(-\alpha)}(-\alpha)}{k !} y^{(k+\alpha)}(x) h^{k}+O\left(h^{m}\right)$,

where $y^{(k+\alpha)}(x)$ is the Caputo derivative of order $k+\alpha$ of the function $y$

$$
y^{(k+\alpha)}(x)=\frac{1}{\Gamma(1-\alpha)} \int_{0}^{x} \frac{y^{(k+1)}(t)}{(x-t)^{\alpha}} d t .
$$

The asymptotic expansion formula of the Grünwald-Letnikov approximation is obtained from the series expansion of the Fourier transform of the approximation. Lubich (1986) constructs higher-order approximations of the fractional derivative which are derived from the Fourier transform of the approximation and the properties of the generating function. Second-order and trird-order approximations of the Caputo and Riemann-Louville fractional derivatives related to the Grünwald-Letnikov difference approximation and their applications for numerical solution of fractional differential equations are studied in (Tadjeran et al. 2006; Dimitrov 2014; Vong and Wang 2014; Tian et al. 2015; Gao et al. 2015; Ren and Wang 2017). High-order approximations of the fractional derivative whose generating function is related to the generating function of the Grünwald-Letnikov approximation are discussed in (Chen and Deng 2014, Ding and Li 2016, 2017). Another approach for constructing approximations of the Caputo derivative uses Lagrange interpolation of the function and computation of the fractional integrals on the stencils of the grid. The L1 approximation is an important and commonly used approximation of the Caputo derivative (Zhuang and Liu 2006; Lin and Xu 2007; Jin et al. 2016).

$$
\frac{1}{h^{\alpha}} \sum_{n=0}^{N} \sigma_{n}^{(\alpha)} y(x-n h)=y^{(\alpha)}(x)+O\left(h^{2-\alpha}\right),
$$


where $\sigma_{0}^{(\alpha)}=1 / \Gamma(2-\alpha), \quad \sigma_{N}^{(\alpha)}=\left((N-1)^{1-\alpha}-N^{1-\alpha}\right) / \Gamma(2-\alpha)$ and

$$
\sigma_{k}^{(\alpha)}=\frac{(k-1)^{1-\alpha}-2 k^{1-\alpha}+(k+1)^{1-\alpha}}{\Gamma(2-\alpha)}, \quad(k=1,2, \ldots, N-1) .
$$

When the function $y \in C^{2}[0, x]$, the $\mathrm{L} 1$ approximation of the Caputo derivative has an accuracy $O\left(h^{2-\alpha}\right)$. In (Dimitrov 2016) we obtain the second-order asymptotic expansion formula of the L1 approximation

$$
\frac{1}{h^{\alpha}} \sum_{k=0}^{N} \sigma_{k}^{(\alpha)} y(x-k h)=y^{(\alpha)}(x)+\frac{\zeta(\alpha-1)}{\Gamma(2-\alpha)} y^{\prime \prime}(x) h^{2-\alpha}+O\left(h^{2}\right)
$$

and a second-order approximation of the Caputo derivative

$$
\frac{1}{h^{\alpha}} \sum_{n=0}^{N} \delta_{k}^{(\alpha)} y(x-k h)=y^{(\alpha)}(x)+O\left(h^{2}\right),
$$

where $\delta_{k}^{(\alpha)}=\sigma_{k}^{(\alpha)}$ for $3 \leq k \leq N$ and

$$
\delta_{0}^{(\alpha)}=\sigma_{0}^{(\alpha)}-\frac{\zeta(\alpha-1)}{\Gamma(2-\alpha)}, \delta_{1}^{(\alpha)}=\sigma_{1}^{(\alpha)}+\frac{2 \zeta(\alpha-1)}{\Gamma(2-\alpha)}, \delta_{2}^{(\alpha)}=\sigma_{2}^{(\alpha)}-\frac{\zeta(\alpha-1)}{\Gamma(2-\alpha)} .
$$

When $k>2$, the weights $\delta_{k}^{(\alpha)}$ of approximation (4) are equal to the weights of the L1 approximation and the first three weights are modified with the value of the zeta function at the point $\alpha-1$. The asymptotic expansions of order $2+\alpha$ of the weights of the Grünwald-Letnikov and the L1 approximations of the Caputo derivative are obtained from the binomial series expansion formula and the asymptotic formula for the gamma function:

$$
w_{k}^{(\alpha)} \sim \frac{1}{\Gamma(-\alpha) k^{1+\alpha}}, \quad \sigma_{k}^{(\alpha)} \sim \frac{1}{\Gamma(-\alpha) k^{1+\alpha}} .
$$

The L1 approximation is constructed by approximating the first derivative by its value at the midpoint of an uniform grid. Gao et al. (2014) and Alikhanov (2015) construct L1 -2 and L $2-1_{\sigma}$ approximations of the Caputo derivative which have accuracy $O\left(h^{3-\alpha}\right)$. Higher-order approximations of the Caputo derivative, related to the construction of the L1 approximation are studied in (Li et al. 2011; Yan et al. 2014; Zheng et al. 2017). In the last two decades many of the methods used for numerical solution of ordinary and partial differential equations have been applied for numerical solution of fractional differential equation; which include spline collocations methods (Pedas and Tamme 2014), Petrov-Galerkin spectral methods (Zayernouri and Karniadakis 2014), orthogonal Legandre and Laguerre polynomials (Bhrawy et al. 2015, Ezz-Eldien et al. 2017), Adomian decomposition methods (El-Borai et al. 2015). In the present paper we study the asymptotic properties of the weights of the GrünwaldLetnikov difference approximation and approximations of the Caputo derivative related to the Grünwald-Letnikov approximation. The approximations discussed in the paper are applied for construction of finite-difference schemes 
for numerical solution of ordinary fractional differential equations. The paper is organized as follows. In section 2 and section 3 we construct approximations of the Caputo derivative which are obtained from the shifted Grünwald-Letnikov approximation and the L1 approximation of the Caputo derivative by replacing the weights whose index is greater than $[N / 5]$ with the first two terms of their asymptotic expansions formulas In section 4 we obtain the second-order shifted approximation of the Caputo derivative:

$$
\frac{1}{h^{\alpha}} \sum_{k=0}^{n} \gamma_{k}^{(\alpha)} y_{n-k}=y_{n-\alpha / 2}^{(\alpha)}+O\left(h^{2}\right)
$$

where $\gamma_{k}^{(\alpha)}=(-1)^{k}\left(\begin{array}{c}\alpha \\ k\end{array}\right)$ for $0 \leq k \leq n-2$ and

$$
\begin{gathered}
\gamma_{n-1}^{(\alpha)}=(-1)^{n-2}\left(\begin{array}{l}
\alpha-2 \\
n-2
\end{array}\right) \frac{n-2 \alpha}{1+\alpha-n}+\frac{(n-\alpha / 2)^{1-\alpha}}{\Gamma(2-\alpha)}, \\
\gamma_{n}^{(\alpha)}=(-1)^{n-2}\left(\begin{array}{l}
\alpha-2 \\
n-2
\end{array}\right)-\frac{(n-\alpha / 2)^{1-\alpha}}{\Gamma(2-\alpha)} .
\end{gathered}
$$

Approximation (5) is obtained from the Grünwald-Letnikov approximation by modifying the last two weights. While the Grünwald-Letnikov approximation is a second-order shifted approximation of the Caputo derivative for the functions $y \in C^{2}\left[0, x_{n}\right]$ which satisfy the condition $y(0)=y^{\prime}(0)=0$, approximation (5) is a second-order approximation of the Caputo derivative $y_{n-\alpha / 2}^{(\alpha)}$ for all function $y \in C^{2}\left[0, x_{n}\right]$. In section 5 we derive the expansion formulas of order $2+\alpha$ of the weights $\gamma_{n-1}^{(\alpha)}$ and $\gamma_{n}^{(\alpha)}$ of approximation (5).

\section{Asymptotic expansion formula for binomial coefficients and shifted approximations for the Caputo derivative}

The asymptotic expansion formula for the ratio of gamma functions is studied in (Tricomi and Erdélyi 1951). The ratio of gamma functions satisfies:

$$
\frac{\Gamma(k+t)}{\Gamma(k+s)}=k^{t-s} \sum_{m=0}^{\infty} \frac{(-1)^{m} B_{m}^{(t-s+1)}(t)(s-t)^{(m)}}{m !} \frac{1}{k^{m}} .
$$

The generalized Bernoulli polynomials $B_{m}^{(\alpha)}(x)$ are defined as the coefficients of the series expansion of the function $e^{x t} t^{\alpha} /\left(e^{t}-1\right)^{\alpha}$

$$
e^{x t}\left(\frac{t}{e^{t}-1}\right)^{\alpha}=\sum_{m=0}^{\infty} B_{m}^{(\alpha)}(x) \frac{t^{m}}{m !} .
$$

From (6) with $t:=-\alpha, s:=1$ we obtain the asymptotic expansion formula for the weights of the Grünwald-Letnikov approximation (Elezović 2005)

$$
w_{k}^{(\alpha)}=(-1)^{k}\left(\begin{array}{l}
\alpha \\
k
\end{array}\right)=\frac{1}{\Gamma(-\alpha)} \sum_{m=0}^{\infty} \frac{(-1)^{m} B_{m}^{(-\alpha)}(-\alpha)(\alpha+1)^{(m)}}{m ! k^{m+\alpha+1}}
$$


where $(\alpha+1)^{(m)}$ is the rising factorial

$$
(\alpha+1)^{(m)}=(\alpha+1)(\alpha+2) \cdots(\alpha+m) .
$$

The generalized Bernoulli polynomial $B_{0}^{(-\alpha)}(-\alpha)=1$ and

$$
B_{1}^{(-\alpha)}(-\alpha)=-\frac{\alpha}{2}, B_{2}^{(-\alpha)}(-\alpha)=\frac{1}{12} \alpha(1+3 \alpha), B_{3}^{(-\alpha)}(-\alpha)=-\frac{1}{8} \alpha^{2}(1+\alpha) .
$$

When $\alpha=1$ the generalized Bernoulli polynomials are equal to the Bernoulli polynomials. The gamma function satisfies the identity $\Gamma(x+1)=x \Gamma(x)$ and $(-1)^{m}(\alpha+1)^{(m)} / \Gamma(-\alpha)=1 / \Gamma(-m-\alpha)$. The weights $w_{k}^{(\alpha)}$ of the GrünwaldLetnikov approximation have an asymptotic expansion of order $M+\alpha+2$

$$
w_{k}^{(\alpha)}=(-1)^{k}\left(\begin{array}{l}
\alpha \\
k
\end{array}\right)=\sum_{m=0}^{M} \frac{B_{m}^{(-\alpha)}(-\alpha)}{m ! \Gamma(-m-\alpha)} \frac{1}{k^{m+\alpha+1}}+O\left(\frac{1}{k^{M+\alpha+2}}\right) .
$$

From (7) with $M=2$ we obtain the asymptotic expansion of order $4+\alpha$ of the weights of the Grünwald-Letnikov approximation:

$$
w_{k}^{(\alpha)}=\frac{1}{\Gamma(-\alpha) k^{1+\alpha}}-\frac{\alpha}{2 \Gamma(-1-\alpha) k^{2+\alpha}}+\frac{\alpha(3 \alpha+1)}{24 \Gamma(-2-\alpha) k^{3+\alpha}}+O\left(\frac{1}{k^{4+\alpha}}\right) .
$$

Now we construct a second-order shifted approximation of the Caputo derivative by replacing the weights of the Grünwald-Letnikov approximation which have an index $k>\lceil N / 5\rceil$ with the first two terms of expansion formula (8).

$$
\bar{w}_{k}^{(\alpha)}= \begin{cases}(-1)^{k}\left(\begin{array}{c}
\alpha \\
k
\end{array}\right), & 0 \leq k \leq\lceil N / 5\rceil \\
\frac{1}{\Gamma(-\alpha) k^{1+\alpha}}-\frac{\alpha}{2 \Gamma(-1-\alpha) k^{2+\alpha}}, & \lceil N / 5\rceil+1 \leq k \leq N-1\end{cases}
$$

In Theorem 1 we show that the approximation with weights $\bar{w}_{k}^{(\alpha)}$ is a secondorder shifted approximation of the Caputo derivative.

Theorem 1 Let $y \in C^{2}[0, x]$ and $y(0)=y^{\prime}(0)=0$. Then

$$
\frac{1}{h^{\alpha}} \sum_{k=0}^{N} \bar{w}_{k}^{(\alpha)} y(x-k h)=y^{(\alpha)}(x-\alpha h / 2)+O\left(h^{2}\right) .
$$

Proof Let $M=\max _{t \in[0, x]}|y(t)|$ and $C>0$, such that when $k>\lceil N / 5\rceil$

$$
\left|(-1)^{k}\left(\begin{array}{l}
\alpha \\
k
\end{array}\right)-\left(\frac{1}{\Gamma(-\alpha) k^{1+\alpha}}-\frac{\alpha}{2 \Gamma(-1-\alpha) k^{2+\alpha}}\right)\right|<\frac{C}{k^{3+\alpha}} .
$$


The difference $E_{N}[y(x)]$ between approximation (9) and the Grünwald-Letnikov approximation satisfies the estimate:

$$
\begin{aligned}
E_{N}[y(x)] & =\left|\frac{1}{h^{\alpha}} \sum_{k=0}^{N-1} \bar{w}_{k}^{(\alpha)} y(x-k h)-\frac{1}{h^{\alpha}} \sum_{k=0}^{N-1}(-1)^{k}\left(\begin{array}{l}
\alpha \\
k
\end{array}\right) y(x-k h)\right| \leq \\
\frac{1}{h^{\alpha}} & \sum_{k=\lceil N / 5\rceil+1}^{N-1}\left|\bar{w}_{k}^{(\alpha)}-(-1)^{k}\left(\begin{array}{l}
\alpha \\
k
\end{array}\right)\right||y(x-k h)|<\frac{C M}{h^{\alpha}} \sum_{k=\lceil N / 5\rceil+1}^{N} \frac{1}{k^{3+\alpha}} .
\end{aligned}
$$

The function $1 / x^{3+\alpha}$ is decreasing and

$$
\sum_{k=\lceil N / 5\rceil+1}^{\infty} \frac{1}{k^{3+\alpha}}<\int_{\lceil N / 5\rceil}^{\infty} \frac{1}{x^{3+\alpha}} d x=\left[-\frac{1}{(2+\alpha) x^{2+\alpha}}\right]_{\lceil N / 5\rceil}^{\infty}<\frac{1}{\lceil N / 5\rceil^{2+\alpha}}
$$

Let $C_{1}=C M(5 / x)^{2+\alpha}$. Then

$$
E_{N}[y(x)] \leq \frac{C M}{h^{\alpha}} \frac{1}{(N / 5)^{2+\alpha}}=\frac{C M}{h^{\alpha}} \frac{5^{2+\alpha}}{N^{2+\alpha}}=C_{1} h^{2} .
$$

From the triangle inequality

$$
\begin{aligned}
\left|\frac{1}{h^{\alpha}} \sum_{k=0}^{N-1} \bar{w}_{k}^{(\alpha)} y(x-\alpha h / 2)-y^{(\alpha)}(x-k h)\right| & \\
& \left|A_{N}^{G L}[y(x)]-y^{(\alpha)}(x-\alpha h / 2)\right|+E_{N}[y(x)] .
\end{aligned}
$$

From (11) and (10)

$$
\frac{1}{h^{\alpha}} \sum_{k=0}^{N-1} \bar{w}_{k}^{(\alpha)} y(x-k h)=y^{(\alpha)}(x-\alpha h / 2)+O\left(h^{2}\right) .
$$

The result of Theorem 1 can be generalized to the approximations for the Caputo derivative which are obtained from any approximation by modifying the weights which have an index greater than $\lceil N / p\rceil$ with the first terms of their expansion formulas, where $p$ is a positive number. When the function $y \in C^{2}\left[0, x_{n}\right]$ and satisfies the condition $y(0)=y^{\prime}(0)=0$ the GrünwaldLetnikov approximation has a second-order expansion formula:

$$
\frac{1}{h^{\alpha}} \sum_{k=0}^{n-1}(-1)^{k}\left(\begin{array}{l}
\alpha \\
k
\end{array}\right) y_{n-k}=y_{n}^{(\alpha)}-\frac{\alpha}{2} y_{n}^{(1+\alpha)} h+O\left(h^{2}\right)=y_{n-\alpha / 2}^{(\alpha)}+O\left(h^{2}\right) .
$$

In (Dimitrov 2018) we derive the expansion formula for the right endpoint of the approximation for the Caputo derivative which has weights $k^{-1-\alpha} / \Gamma(-\alpha)$. 
When the function $y \in C^{2}[0, x]$ and satisfies the condition $y(0)=y^{\prime}(0)=0$, the approximation has an asymptotic expansion of order $3-\alpha$

$$
\begin{aligned}
\frac{1}{h^{\alpha}} \sum_{k=1}^{n-1} \frac{y_{n-k}}{\Gamma(-\alpha) k^{1+\alpha}}=y_{n}^{(\alpha)}+ & \frac{\zeta(\alpha+1)}{\Gamma(-\alpha)} y_{n} h^{-\alpha}- \\
& \frac{\zeta(\alpha)}{\Gamma(-\alpha)} y_{n}^{\prime} h^{1-\alpha}+\frac{\zeta(\alpha-1)}{2 \Gamma(-\alpha)} y_{n}^{\prime \prime} h^{2-\alpha}+O\left(h^{3-\alpha}\right) .
\end{aligned}
$$

Expansion formula (12) is obtained by applying formal integration by parts to the fractional integral in the definition of the Caputo derivative and a Fourier transform to the approximation. The expansion formula for the left endpoint of approximation (12) is obtained from the Euler-Mclaurin formula for the function $y(t) /(x-t)^{1+\alpha}$. By substituting $\alpha:=\alpha+1$ in (12) we obtain

$$
\begin{gathered}
\frac{1}{h^{1+\alpha}} \sum_{k=1}^{n-1} \frac{y_{n-k}}{\Gamma(-1-\alpha) k^{2+\alpha}}=y_{n}^{(1+\alpha)}+\frac{\zeta(\alpha+2)}{\Gamma(-1-\alpha)} y_{n} h^{-1-\alpha}- \\
\frac{\zeta(1+\alpha)}{\Gamma(-1-\alpha)} y_{n}^{\prime} h^{-\alpha}+\frac{\zeta(\alpha)}{2 \Gamma(-1-\alpha)} y_{n}^{\prime \prime} h^{1-\alpha}+O\left(h^{2-\alpha}\right) \\
\frac{1}{h^{\alpha}} \sum_{k=1}^{n-1} \frac{y_{n-k}}{\Gamma(-1-\alpha) k^{2+\alpha}}=h y_{n}^{(1+\alpha)}+\frac{\zeta(\alpha+2)}{\Gamma(-1-\alpha)} y_{n} h^{-\alpha}- \\
\frac{\zeta(1+\alpha)}{\Gamma(-1-\alpha)} y_{n}^{\prime} h^{1-\alpha}+\frac{\zeta(\alpha)}{2 \Gamma(-1-\alpha)} y_{n}^{\prime \prime} h^{2-\alpha}+O\left(h^{3-\alpha}\right)
\end{gathered}
$$

By multiplying (13) by $-\alpha / 2$ and adding to (12) we obtain

$$
\begin{aligned}
& \frac{1}{h^{\alpha}} \sum_{k=1}^{n-1}\left(\frac{1}{\Gamma(-\alpha) k^{1+\alpha}}-\frac{\alpha}{2 \Gamma(-1-\alpha) k^{2+\alpha}}\right) y_{n-k}=y_{n}^{(\alpha)}-\frac{\alpha h}{2} y_{n}^{(1+\alpha)}+ \\
& \left(\frac{\zeta(\alpha+1)}{\Gamma(-\alpha)}-\frac{\alpha \zeta(\alpha+2)}{2 \Gamma(-1-\alpha)}\right) y_{n} h^{-\alpha}-\left(\frac{\zeta(\alpha)}{\Gamma(-\alpha)}-\frac{\alpha \zeta(\alpha+1)}{2 \Gamma(-1-\alpha)}\right) y_{n}^{\prime} h^{1-\alpha}+ \\
& \frac{1}{2}\left(\frac{\zeta(\alpha-1)}{\Gamma(-\alpha)}-\frac{\alpha \zeta(\alpha)}{2 \Gamma(-1-\alpha)}\right) y_{n}^{\prime \prime} h^{2-\alpha}+O\left(h^{3-\alpha}\right)
\end{aligned}
$$

By substituting $y_{n}^{\prime}=\left(y_{n}-y_{n-1}\right) / h+O(h)$ in (14) we obtain

$$
\begin{gathered}
\frac{1}{h^{\alpha}} \sum_{k=1}^{n-1}\left(\frac{1}{\Gamma(-\alpha) k^{1+\alpha}}-\frac{\alpha}{2 \Gamma(-1-\alpha) k^{2+\alpha}}\right) y_{n-k}=y_{n}^{(\alpha)}-\frac{\alpha h}{2} y_{n}^{(1+\alpha)}- \\
\quad \frac{1}{h^{\alpha}}\left(\frac{\zeta(\alpha)}{\Gamma(-\alpha)}-\frac{\alpha \zeta(\alpha+1)}{2 \Gamma(-1-\alpha)}\right)\left(y_{n}-y_{n-1}\right)+ \\
\quad \frac{1}{h^{\alpha}}\left(\frac{\zeta(\alpha+1)}{\Gamma(-\alpha)}-\frac{\alpha \zeta(\alpha+2)}{2 \Gamma(-1-\alpha)}\right) y_{n}+O\left(h^{2-\alpha}\right)
\end{gathered}
$$


From (15) we obtain the shifted approximation for the Caputo derivative of order $2-\alpha$ :

$$
\frac{1}{h^{\alpha}} \sum_{k=0}^{n-1} \widetilde{w}_{k}^{(\alpha)} y_{n-k}=y_{n}^{(\alpha)}-\frac{\alpha}{2} y_{n}^{(1+\alpha)} h+O\left(h^{2-\alpha}\right)=y_{n-\alpha / 2}^{(\alpha)}+O\left(h^{2-\alpha}\right) .
$$

where

$$
\begin{gathered}
\widetilde{w}_{0}^{(\alpha)}=\frac{1}{\Gamma(-\alpha)}\left(\zeta(\alpha)+\frac{1}{2}(\alpha-1)(\alpha+2) \zeta(\alpha+1)-\frac{1}{2} \alpha(\alpha+1) \zeta(\alpha+2)\right) \\
\widetilde{w}_{1}^{(\alpha)}=\frac{1}{\Gamma(-\alpha)}\left(\frac{1}{2}\left(\alpha^{2}+\alpha+2\right)-\zeta(\alpha)-\frac{1}{2} \alpha(\alpha+1) \zeta(\alpha+1)\right) \\
\widetilde{w}_{k}^{(\alpha)}=\frac{1}{\Gamma(-\alpha) k^{1+\alpha}}-\frac{\alpha}{2 \Gamma(-1-\alpha) k^{2+\alpha}}, \quad(k=2, \ldots, n-1) .
\end{gathered}
$$

When the function $y \in C^{2}\left[0, x_{n}\right]$ and satisfies the condition $y(0)=y^{\prime}(0)=0$ approximation (16) an approximation for $y_{n-\alpha / 2}^{(\alpha)}$ with an accuracy $O\left(h^{2-\alpha}\right)$. The weights of approximation (16) are equal to the first two terms in the asymptotic expansion formula of the weights of the Grünwald-Letnikov approximation $(-1)^{k}\left(\begin{array}{l}\alpha \\ k\end{array}\right)$, when $k \geq 2$. The first two terms of the second-order expansion of the Grünwald-Letnikov approximation and the expansion formula of order $2-\alpha$ of approximation (16) are equal.

By substituting $y_{n}^{\prime \prime}=\frac{1}{h^{2}}\left(y_{n}-2 y_{n-1}+y_{n-2}\right) / h^{2}+O(h)$ and

$$
y_{n}^{\prime}=\frac{1}{h}\left(\frac{3}{2} y_{n}-2 y_{n-1}+\frac{1}{2} y_{n-2}\right)+O\left(h^{2}\right),
$$

in (14) we obtain the shifted approximation for the Caputo derivative:

$$
\frac{1}{h^{\alpha}} \sum_{k=0}^{n-1} \widehat{w}_{k}^{(\alpha)} y_{n-k}=y_{n-\alpha / 2}^{(\alpha)}+O\left(h^{2}\right)=y_{n}^{(\alpha)}-\frac{\alpha}{2} y_{n}^{(1+\alpha)} h+O\left(h^{3-\alpha}\right),
$$

where

$$
\begin{aligned}
& \widehat{w}_{0}^{(\alpha)}=-\frac{1}{4 \Gamma(-\alpha)}(2 \zeta(-1+\alpha)+(\alpha+3)(\alpha-2) \zeta(\alpha)- \\
& \left.\left(3 \alpha^{2}+3 \alpha-4\right) \zeta(\alpha+1)+2 \alpha(\alpha+1) \zeta(2+\alpha)\right), \\
& \widehat{w}_{1}^{(\alpha)}=\frac{1}{2 \Gamma(-\alpha)}\left(2+\alpha+\alpha^{2}+2 \zeta(-1+\alpha)+\right. \\
& \left.\quad\left(\alpha+\alpha^{2}-4\right) \zeta(\alpha)-2 \alpha(\alpha+1) \zeta(1+\alpha)\right), \\
& \widehat{w}_{2}^{(\alpha)}=\frac{1}{4 \Gamma(-\alpha)}\left(\frac{4+\alpha+\alpha^{2}}{2^{1+\alpha}-2 \zeta(-1+\alpha)-}\right. \\
& \left.\quad\left(\alpha^{2}+\alpha-2\right) \zeta(\alpha)+\alpha(1+\alpha) \zeta(1+\alpha)\right),
\end{aligned}
$$




$$
\widehat{w}_{k}^{(\alpha)}=\frac{1}{\Gamma(-\alpha) k^{1+\alpha}}-\frac{\alpha}{2 \Gamma(-1-\alpha) k^{2+\alpha}}, \quad(k=3, \ldots, n-1) .
$$

When the function $y \in C^{2}\left[0, x_{n}\right]$ and satisfies the condition $y(0)=y^{\prime}(0)=0$ approximation (17) is a second-order approximation for the Caputo derivative at the point $x_{n}-\alpha h / 2$. The weights of approximation (17) are equal to the first two terms in the expansion formula of $(-1)^{k}\left(\begin{array}{l}\alpha \\ k\end{array}\right)$ for $k \geq 3$. The first two terms of the second-order asymptotic expansion of the Grünwald-Letnikov approximation are equal to the first two terms of the expansion of order $3-\alpha$ of approximation (17).

\section{Expansion formula for the weights of the L1 approximation}

From the binomial formula:

$$
\begin{gathered}
(k+1)^{1-\alpha}=k^{1-\alpha}\left(1+\frac{1}{k}\right)^{1-\alpha}=n^{1-\alpha}\left(\sum_{m=0}^{5}\left(\begin{array}{c}
1-\alpha \\
m
\end{array}\right) \frac{1}{k^{m}}+O\left(\frac{1}{k^{6}}\right)\right), \\
(k+1)^{1-\alpha}=k^{1-\alpha}+\sum_{m=1}^{5}\left(\begin{array}{c}
1-\alpha \\
m
\end{array}\right) \frac{1}{k^{m+\alpha-1}}+O\left(\frac{1}{k^{5+\alpha}}\right) .
\end{gathered}
$$

Similarly

$$
(k-1)^{1-\alpha}=k^{1-\alpha}+\sum_{m=1}^{5}(-1)^{m}\left(\begin{array}{c}
1-\alpha \\
m
\end{array}\right) \frac{1}{k^{m+\alpha-1}}+O\left(\frac{1}{k^{5+\alpha}}\right) .
$$

From (18) and (19)

$$
\begin{gathered}
(k-1)^{1-\alpha}-2 k^{1-\alpha}+(k+1)^{1-\alpha}=2\left(\begin{array}{c}
1-\alpha \\
2
\end{array}\right) \frac{1}{k^{1+\alpha}}+2\left(\begin{array}{c}
1-\alpha \\
4
\end{array}\right) \frac{1}{k^{3+\alpha}}+O\left(\frac{1}{k^{5+\alpha}}\right), \\
\Gamma(2-\alpha) \sigma_{k}^{(\alpha)}=-\frac{(1-\alpha) \alpha}{k^{1+\alpha}}+\frac{(1-\alpha)(-\alpha)(-\alpha-1)(-\alpha-2)}{12 k^{3+\alpha}}+O\left(\frac{1}{k^{5+\alpha}}\right) .
\end{gathered}
$$

The weights $\sigma_{k}^{(\alpha)}$ of the L1 approximation have an asymptotic expansion of order $5+\alpha$, for $1 \leq k \leq n-1$

$$
\sigma_{k}^{(\alpha)}=\frac{1}{\Gamma(-\alpha) k^{1+\alpha}}+\frac{1}{12 \Gamma(-2-\alpha) k^{3+\alpha}}+O\left(\frac{1}{k^{5+\alpha}}\right) .
$$

The last weight $\sigma_{n}^{(\alpha)}$ of the L1 approximation (2) satisfies

$$
\Gamma(2-\alpha) \sigma_{n}^{(\alpha)}=(n-1)^{1-\alpha}-n^{1-\alpha} .
$$

From (19)

$$
\Gamma(2-\alpha) \sigma_{n}^{(\alpha)}=-\left(\begin{array}{c}
1-\alpha \\
1
\end{array}\right) \frac{1}{n^{\alpha}}+\left(\begin{array}{c}
1-\alpha \\
2
\end{array}\right) \frac{1}{n^{1+\alpha}}-\left(\begin{array}{c}
1-\alpha \\
3
\end{array}\right) \frac{1}{n^{2+\alpha}}+O\left(\frac{1}{n^{3+\alpha}}\right),
$$




$$
\sigma_{n}^{(\alpha)}=-\frac{1}{\Gamma(1-\alpha) n^{\alpha}}+\frac{1}{2 \Gamma(-\alpha) n^{1+\alpha}}-\frac{1}{6 \Gamma(-1-\alpha) n^{2+\alpha}}+O\left(\frac{1}{n^{3+\alpha}}\right) .
$$

Similarly to the construction of approximation (9) we use asymptotic expansion formulas (20) and (21) to obtain approximations of the Caputo derivative by modifying the weights $\sigma_{k}^{(\alpha)}$ of the L1 approximation and the second order approximation (4) when $k>\lceil N / 5\rceil$.

$$
\frac{1}{h^{\alpha}} \sum_{k=0}^{n} \bar{\sigma}_{k}^{(\alpha)} y_{n-k}=y_{n}^{(\alpha)}+O\left(h^{2-\alpha}\right)
$$

where $\bar{\sigma}_{0}^{(\alpha)}=1 / \Gamma(2-\alpha)$ and

$$
\bar{\sigma}_{k}^{(\alpha)}=\left\{\begin{array}{l}
\frac{(k-1)^{1-\alpha}-2 k^{1-\alpha}+(k+1)^{1-\alpha}}{\Gamma(2-\alpha)}, 1 \leq k \leq\lceil N / 5\rceil, \\
\frac{1}{\Gamma(-\alpha) k^{1+\alpha}}+\frac{\alpha}{12 \Gamma(-2-\alpha) k^{3+\alpha}},\lceil N / 5\rceil<k \leq N-1 .
\end{array}\right.
$$

When $n \leq\lceil N / 5\rceil$ the last weight $\bar{\sigma}_{n}^{(\alpha)}$ of approximation (22) is equal to the last weight $\sigma_{n}^{(\alpha)}$ of the L1 approximation. When $n>\lceil N / 5\rceil$ the value of the weight $\bar{\sigma}_{n}^{(\alpha)}$ is equal to the terms of expansion formula (21) of $\sigma_{n}^{(\alpha)}$.

$$
\bar{\sigma}_{n}^{(\alpha)}= \begin{cases}\sigma_{n}^{(\alpha)}=\frac{(n-1)^{1-\alpha}-n^{1-\alpha}}{\Gamma(2-\alpha)}, & n \leq\lceil N / 5\rceil, \\ -\frac{n^{-\alpha}}{\Gamma(1-\alpha)}+\frac{n^{-\alpha-1}}{2 \Gamma(-\alpha)}-\frac{n^{-\alpha-2}}{6 \Gamma(-1-\alpha)}, & n>\lceil N / 5\rceil .\end{cases}
$$

From approximation (4) we obtain the second-order approximation of the Caputo derivative

$$
\frac{1}{h^{\alpha}} \sum_{k=0}^{n} \bar{\delta}_{k}^{(\alpha)} y_{n-k}=y_{n}^{(\alpha)}+O\left(h^{2}\right)
$$

where the weights $\bar{\delta}_{k}^{(\alpha)}=\bar{\sigma}_{k}^{(\alpha)}$ for $k \geq 3$ are defined with (23), (24) and $\bar{\delta}_{k}^{(\alpha)}=\delta_{k}^{(\alpha)}$ for $k=0,1,2: \bar{\delta}_{0}^{(\alpha)}=(1-\zeta(\alpha-1)) / \Gamma(2-\alpha)$,

$$
\bar{\delta}_{1}^{(\alpha)}=\frac{2-2^{1-\alpha}+2 \zeta(\alpha-1)}{\Gamma(2-\alpha)}, \bar{\delta}_{2}^{(\alpha)}=\frac{3^{1-\alpha}-2^{-\alpha}+1-\zeta(\alpha-1)}{\Gamma(2-\alpha)} .
$$

When the function $y \in C^{2}\left[0, x_{n}\right]$, approximations (22) and (25) have accuracy $O\left(h^{2-\alpha}\right)$ and $O\left(h^{2}\right)$. The proof is similar to the proof of Theorem 1 .

The fractional integral of order $\alpha>0$ is defined as

$$
I^{\alpha} y(x)=y^{(-\alpha)}(x)=\int_{0}^{x}(x-t)^{\alpha-1} y(t) d t
$$


The fractional integral $I^{\alpha} y(x)$ is the fractional derivative of the function $y(x)$ of order $-\alpha$. The Riemann sum approximation of the fractional integral has an expansion of order $1+\alpha$

$$
h^{\alpha} \sum_{k=1}^{n-1} \frac{y_{n-k}}{\Gamma(\alpha) k^{1-\alpha}}=y_{n}^{(-\alpha)}+\frac{\zeta(1-\alpha)}{\Gamma(\alpha)} y_{n} h^{\alpha}+O\left(h^{1+\alpha}\right),
$$

when $y(0)=0$. From the approximations discussed in the paper and other approximations for the fractional derivatives and integrals we observe that the weights of the approximation $h^{-\alpha} \sum_{k=0}^{n} w_{k}^{(\alpha)} y(x-k h)$ of the fractional derivative $y^{(\alpha)}(x)$ satisfy $w_{k}^{(\alpha)} \sim \frac{c_{k}}{k^{1+\alpha}}$ and weights of the approximations of the definite integral satisfy $w_{k}^{(-1)} \sim c_{k}$. This property holds for the trapezoidal approximation, Simpson's approximation, and the quadrature formulas discussed in (Dimitrov, Miryanov and Todorov 2017).

\section{Shifted Grünwald-Letnikov difference approximation}

In this section we use the method from (Dimitrov 2018) to derive an approximation (5) for the Caputo derivative, which is obtained from the GrünwaldLetnikov approximation by modifying the last two weights. Approximation (5) is a second-order shifted approximation for the Caputo derivative $y_{n-\alpha / 2}^{(\alpha)}$ for all functions $y \in C^{2}\left[0, x_{n}\right]$. Let $y_{0}=y(0), y_{0}^{\prime}=y^{\prime}(0)$ and

$$
y(x)=y_{0}+y_{0}^{\prime} x+z(x) .
$$

The function $z(x)$ satisfies the condition $z(0)=z^{\prime}(0)=0$.

$$
\begin{gathered}
A_{N}^{G L}[y(x)]=y_{0} A_{N}^{G L}[1]+y_{0}^{\prime} A_{N}^{G L}[x]+A_{N}^{G L}[z(x)], \\
A_{N}^{G L}[y(x)]=y_{0} A_{N}^{G L}[1]+y_{0}^{\prime} A_{N}^{G L}[x]+z^{(\alpha)}(x-\alpha h / 2)+O\left(h^{2}\right) .
\end{gathered}
$$

The Caputo derivative of the functions $y$ and $z$ satisfies

$$
y^{(\alpha)}(x-\alpha h / 2)=\frac{y_{0}^{\prime}}{\Gamma(2-\alpha)}(x-\alpha h / 2)^{1-\alpha}+z^{(\alpha)}(x-\alpha h / 2) .
$$

Then

$$
\begin{gathered}
A_{N}^{G L}[y(x)]=y_{0} A_{h}^{G L}[1]+y_{0}^{\prime} A_{h}^{G L}[x]-\frac{y_{0}^{\prime}(x-\alpha h / 2)^{1-\alpha}}{\Gamma(2-\alpha)}+y^{(\alpha)}(x-\alpha h / 2)+O\left(h^{2}\right), \\
A_{N}^{G L}[y(x)]=y_{0} A_{N}^{G L}[1]+\left(A_{N}^{G L}[x]-\frac{(x-\alpha h / 2)^{1-\alpha}}{\Gamma(2-\alpha)}\right) y_{0}^{\prime}+ \\
y^{(\alpha)}(x-\alpha h / 2)+O\left(h^{2}\right) .
\end{gathered}
$$


Denote $W_{N}^{0}=h^{\alpha} A_{N}^{G L}[1]=\sum_{k=0}^{N-1} w_{k}^{(\alpha)}$ and

$$
\begin{gathered}
W_{N}^{1}=\frac{1}{h^{1-\alpha}}\left(A_{h}^{G L}[x]-\frac{(x-\alpha h / 2)^{1-\alpha}}{\Gamma(2-\alpha)}\right) \\
W_{N}^{1}=\frac{1}{h^{1-\alpha}}\left(\frac{1}{h^{\alpha}} \sum_{k=0}^{N-1} w_{k}^{(\alpha)}(x-k h)-\frac{h^{1-\alpha}}{\Gamma(2-\alpha)}(N-\alpha / 2)^{1-\alpha}\right), \\
W_{N}^{1}=N W_{N}^{0}-\sum_{k=0}^{N-1} k w_{k}^{(\alpha)}-\frac{(N-\alpha / 2)^{1-\alpha}}{\Gamma(2-\alpha)} .
\end{gathered}
$$

In Lemma 2 we show that $W_{N}^{1}=O\left(h^{1+\alpha}\right)$. From (26), the Grünwald-Letnikov approximation satisfies:

$$
A_{N}^{G L}[y(x)]=y^{(\alpha)}(x-\alpha h / 2)+\frac{1}{h^{\alpha}}\left(W_{N}^{0} y_{0}+W_{N}^{1} y_{0}^{\prime} h\right)+O\left(h^{2}\right) .
$$

By substituting $h y_{0}^{\prime}=y_{1}-y_{0}+O\left(h^{2}\right)$ we obtain

$$
A_{N}^{G L}[y(x)]=y^{(\alpha)}(x-\alpha h / 2)+\frac{1}{h^{\alpha}}\left(W_{N}^{0} y_{0}+W_{N}^{1}\left(y_{1}-y_{0}\right)\right)+O\left(h^{2}\right) .
$$

Denote by $\bar{A}_{N}^{G L}[y(x)]$ the approximation of the Caputo derivative

$$
\bar{A}_{N}^{G L}[y(x)]=A_{N}^{G L}[y(x)]-\frac{1}{h^{\alpha}}\left(W_{N}^{0} y_{0}+W_{N}^{1}\left(y_{1}-y_{0}\right)\right) .
$$

Approximation $\bar{A}_{N}^{G L}[y(x)]$ is obtained from the Grünwald-Letnikov approximation by modifying the last two weights.

$$
\bar{A}_{N}^{G L}[y(x)]=\frac{1}{h^{\alpha}} \sum_{k=0}^{N} \gamma_{k}^{(\alpha)} y(x-k h)=y^{(\alpha)}(x-\alpha h / 2)+O\left(h^{2}\right),
$$

where $\gamma_{k}^{(\alpha)}=w_{k}^{(\alpha)}=(-1)^{k}\left(\begin{array}{l}\alpha \\ k\end{array}\right)$ for $k=0,1, \ldots, N-2$ and

$$
\begin{gathered}
\gamma_{N-1}^{(\alpha)}=w_{N-1}^{(\alpha)}-W_{N}^{1}=(-1)^{N-1}\left(\begin{array}{c}
\alpha \\
N-1
\end{array}\right)-\sum_{k=0}^{N-1}(N-k) w_{k}^{(\alpha)}+\frac{(N-\alpha / 2)^{1-\alpha}}{\Gamma(2-\alpha)}, \\
\gamma_{N-1}^{(\alpha)}=-\sum_{k=0}^{N-2}(-1)^{k}(N-k)\left(\begin{array}{l}
\alpha \\
k
\end{array}\right)+\frac{(N-\alpha / 2)^{1-\alpha}}{\Gamma(2-\alpha)} \\
\gamma_{N}^{(\alpha)}=W_{N}^{1}-W_{N}^{0}=\sum_{k=0}^{N-2}(N-k-1) w_{k}^{(\alpha)}-\frac{(N-\alpha / 2)^{1-\alpha}}{\Gamma(2-\alpha)}-w_{N-1}^{(\alpha)} .
\end{gathered}
$$

By induction we can show that the weights of the Grünwald-Letnikov approximation satisfy the identities (Podlubny 1999)

$$
W_{N}^{0}=\sum_{k=0}^{N-1} w_{k}^{(\alpha)}=\sum_{k=0}^{N-1}(-1)^{k}\left(\begin{array}{l}
\alpha \\
k
\end{array}\right)=(-1)^{N-1}\left(\begin{array}{c}
\alpha-1 \\
N-1
\end{array}\right)=w_{N-1}^{(\alpha-1)}
$$




$$
\sum_{k=1}^{N-1}(-1)^{k} k\left(\begin{array}{l}
\alpha \\
k
\end{array}\right)=-\alpha \sum_{k=1}^{N-1}(-1)^{k-1}\left(\begin{array}{c}
\alpha-1 \\
k-1
\end{array}\right)=-\alpha w_{N-2}^{(\alpha-2)} .
$$

From (27), 281) and (29)

$$
\begin{aligned}
& W_{N}^{1}=N w_{N-1}^{(\alpha-1)}+\alpha w_{N-2}^{(\alpha-2)}-\frac{(N-\alpha / 2)^{1-\alpha}}{\Gamma(2-\alpha)}, \\
& N w_{N-1}^{(\alpha-1)}+\alpha w_{N-2}^{(\alpha-2)}=N(-1)^{N-1}\left(\begin{array}{c}
\alpha-1 \\
N-1
\end{array}\right)+\alpha(-1)^{N-2}\left(\begin{array}{c}
\alpha-2 \\
N-2
\end{array}\right)= \\
& (-1)^{N-2}\left(-\frac{N(\alpha-1)}{N-1}\left(\begin{array}{c}
\alpha-2 \\
N-2
\end{array}\right)+\alpha\left(\begin{array}{c}
\alpha-2 \\
N-2
\end{array}\right)\right)= \\
& (-1)^{N-2}\left(\begin{array}{c}
\alpha-2 \\
N-2
\end{array}\right) \frac{N-\alpha}{N-1}=w_{N-1}^{(\alpha-2)} \text {. }
\end{aligned}
$$

Hence

$$
W_{N}^{1}=w_{N-1}^{(\alpha-2)}-\frac{(N-\alpha / 2)^{1-\alpha}}{\Gamma(2-\alpha)} .
$$

The weight $\gamma_{N}^{(\alpha)}$ of satisfies

$$
\gamma_{N}^{(\alpha)}=W_{N}^{1}-W_{N}^{0}=w_{N-1}^{(\alpha-2)}-w_{N-1}^{(\alpha-1)}-\frac{(N-\alpha / 2)^{1-\alpha}}{\Gamma(2-\alpha)} .
$$

From the properties of the binomial coefficients

$$
w_{N-1}^{(\alpha-2)}-w_{N-1}^{(\alpha-1)}=(-1)^{N-1}\left(\left(\begin{array}{c}
\alpha-2 \\
N-1
\end{array}\right)-\left(\begin{array}{c}
\alpha-1 \\
N-1
\end{array}\right)\right)=(-1)^{N-2}\left(\begin{array}{c}
\alpha-2 \\
N-2
\end{array}\right) .
$$

Hence

$$
\gamma_{N}^{(\alpha)}=w_{N-2}^{(\alpha-2)}-\frac{(N-\alpha / 2)^{1-\alpha}}{\Gamma(2-\alpha)}
$$

The weight $\gamma_{N-1}^{(\alpha)}$ satisfies

$$
\begin{gathered}
\gamma_{N-1}^{(\alpha)}=w_{N-1}^{(\alpha)}-W_{N}^{1}=w_{N-1}^{(\alpha)}-w_{N-1}^{(\alpha-2)}+\frac{(N-\alpha / 2)^{1-\alpha}}{\Gamma(2-\alpha)}, \\
w_{N-1}^{(\alpha)}-w_{N-1}^{(\alpha-2)}=(-1)^{N-1}\left(\left(\begin{array}{c}
\alpha \\
N-1
\end{array}\right)-\left(\begin{array}{c}
\alpha-2 \\
N-1
\end{array}\right)\right)= \\
(-1)^{N-1}\left(\left(\begin{array}{c}
\alpha-2 \\
N-2
\end{array}\right) \frac{\alpha(\alpha-1)}{(N-1)(\alpha-N+1)}-\left(\begin{array}{c}
\alpha-2 \\
N-1
\end{array}\right) \frac{\alpha-N}{N-1}\right)= \\
(-1)^{N-2}\left(\begin{array}{c}
\alpha-2 \\
N-2
\end{array}\right) \frac{N-2 \alpha}{1+\alpha-N} .
\end{gathered}
$$

Hence

$$
\gamma_{N-1}^{(\alpha)}=\frac{N-2 \alpha}{1+\alpha-N} w_{N-2}^{(\alpha-2)}+\frac{(N-\alpha / 2)^{1-\alpha}}{\Gamma(2-\alpha)} .
$$


Approximation $\bar{A}_{n}^{G L}[y(x)]$ is a second-order shifted approximation for the Caputo derivative, when the function $y \in C^{2}\left[0, x_{n}\right]$ :

$$
\frac{1}{h^{\alpha}} \sum_{k=0}^{n} \gamma_{k}^{(\alpha)} y_{n-k}=y_{n-\alpha / 2}^{(\alpha)}+O\left(h^{2}\right)
$$

where $\gamma_{k}^{(\alpha)}=w_{k}^{(\alpha)}=(-1)^{k}\left(\begin{array}{l}\alpha \\ k\end{array}\right)$ for $0 \leq k \leq n-2$ and

$$
\gamma_{n-1}^{(\alpha)}=\frac{n-2 \alpha}{1+\alpha-n} w_{n-2}^{(\alpha-2)}+\frac{(n-\alpha / 2)^{1-\alpha}}{\Gamma(2-\alpha)}, \gamma_{n}^{(\alpha)}=w_{n-2}^{(\alpha-2)}-\frac{(n-\alpha / 2)^{1-\alpha}}{\Gamma(2-\alpha)} .
$$

\section{Asymptotic expansion formulas}

In this section we obtain the asymptotic expansions of $W_{n}^{0}=w_{n-1}^{(\alpha-1)}, W_{n}^{1}$ and the last two weights $\gamma_{n-1}^{(\alpha)}$ and $\gamma_{n}^{(\alpha)}$ of approximation (30), and we construct an approximation of the Caputo derivative by modifying the weights of approximation (30) which have an index greater than $\lceil N / 5\rceil$ with the first terms of their asymptotic expansions.

\section{Lemma 2}

$$
\begin{gathered}
W_{n}^{0}=\frac{1}{\Gamma(1-\alpha) n^{\alpha}}-\frac{\alpha+1}{2 \Gamma(-\alpha) n^{1+\alpha}}+\frac{(2+\alpha)(1+3 \alpha)}{24 \Gamma(-1-\alpha) n^{2+\alpha}}+O\left(\frac{1}{n^{3+\alpha}}\right) . \\
W_{n}^{1}=\frac{\alpha-2}{24 \Gamma(-\alpha) n^{1+\alpha}}+O\left(\frac{1}{n^{2+\alpha}}\right) .
\end{gathered}
$$

Proof From expansion formula (8) for $w_{n}^{(\alpha-1)}$ with $\alpha:=\alpha-1$

$$
\begin{aligned}
w_{n-1}^{(\alpha-1)}=\frac{1}{\Gamma(1-\alpha)(n-1)^{\alpha}}-\frac{\alpha-1}{2 \Gamma(-\alpha)(n-1)^{1+\alpha}}+ & \\
& \frac{(\alpha-1)(3 \alpha-2)}{24 \Gamma(-1-\alpha) n^{2+\alpha}}+O\left(\frac{1}{n^{3+\alpha}}\right) .
\end{aligned}
$$

From the binomial formula

$$
\frac{1}{(n-1)^{\alpha}}=n^{-\alpha}\left(1-\frac{1}{n}\right)^{-\alpha}=\frac{1}{n^{\alpha}}+\frac{\alpha}{n^{1+\alpha}}+\frac{\alpha(\alpha+1) \alpha}{2 n^{2+\alpha}}+O\left(\frac{1}{n^{3+\alpha}}\right) .
$$

By substituting $\alpha:=\alpha+1$ and $\alpha:=\alpha+2$ in (32) we obtain

$\frac{1}{(n-1)^{1+\alpha}}=\frac{1}{n^{1+\alpha}}+\frac{1+\alpha}{n^{2+\alpha}}+O\left(\frac{1}{n^{3+\alpha}}\right), \frac{1}{(n-1)^{2+\alpha}}=\frac{1}{n^{2+\alpha}}+O\left(\frac{1}{n^{3+\alpha}}\right)$.

From (31), (32) and (33) the weight $w_{n-1}^{(\alpha-1)}=W_{n}^{0}$ has an asymptotic expansion $w_{n-1}^{(\alpha-1)}=\frac{1}{\Gamma(1-\alpha) n^{\alpha}}+\left(\frac{\alpha}{\Gamma(1-\alpha)}-\frac{\alpha-1}{2 \Gamma(-\alpha)}\right) \frac{1}{n^{1+\alpha}}+\frac{S}{n^{2+\alpha}}+O\left(\frac{1}{n^{3+\alpha}}\right)$, 
where

$$
S=-\frac{\alpha(\alpha+1)}{2 \Gamma(1-\alpha)}-\frac{(\alpha-1)(\alpha+1)}{2 \Gamma(-\alpha)}-\frac{(\alpha+1)(2+\alpha)}{24 \Gamma(-1-\alpha)}=\frac{(\alpha+2)(3 \alpha+1)}{24 \Gamma(-1-\alpha)}
$$

Hence

$$
w_{n-1}^{(\alpha-1)}=\frac{1}{\Gamma(1-\alpha) n^{\alpha}}-\frac{1+\alpha}{2 \Gamma(-\alpha) n^{1+\alpha}}+\frac{(\alpha+2)(3 \alpha+1)}{24 \Gamma(-1-\alpha) n^{2+\alpha}}+O\left(\frac{1}{n^{3+\alpha}}\right) .
$$

From the binomial formula

$$
\begin{aligned}
& \left(n-\frac{\alpha}{2}\right)^{1-\alpha}=n^{1-\alpha}\left(1-\frac{\alpha}{2 n}\right)^{1-\alpha}=n^{1-\alpha}\left(\sum_{k=0}^{2}\left(\begin{array}{c}
1-\alpha \\
k
\end{array}\right)\left(\frac{\alpha}{2 n}\right)^{k}+O\left(\frac{1}{n^{3}}\right)\right), \\
& \frac{(n-\alpha / 2)^{1-\alpha}}{\Gamma(2-\alpha)}=\frac{1}{\Gamma(2-\alpha) n^{\alpha-1}}+\frac{1}{2 \Gamma(-\alpha) n^{\alpha}}+\frac{\alpha^{2}}{8 \Gamma(-\alpha) n^{\alpha+1}}+O\left(\frac{1}{n^{2+\alpha}}\right) .
\end{aligned}
$$

From (34) with $\alpha:=\alpha-1$

$$
w_{n-1}^{(\alpha-2)}=\frac{1}{\Gamma(2-\alpha) n^{\alpha-1}}-\frac{\alpha}{2 \Gamma(1-\alpha) n^{\alpha}}+\frac{(\alpha+1)(3 \alpha-2)}{24 \Gamma(-\alpha) n^{1+\alpha}}+O\left(\frac{1}{n^{2+\alpha}}\right) .
$$

Hence

$$
W_{n}^{1}=w_{n-1}^{(\alpha-2)}-\frac{(n-\alpha / 2)^{1-\alpha}}{\Gamma(2-\alpha)}=\frac{\alpha-2}{24 \Gamma(-\alpha) n^{1+\alpha}}+O\left(\frac{1}{n^{2+\alpha}}\right) .
$$

In the Lemma 3 we use the expansion formulas of $W_{n}^{0}$ and $W_{n}^{1}$ to obtain the expansions of order $2+\alpha$ of the weights $\gamma_{n-1}^{(\alpha)}$ and $\gamma_{n}^{(\alpha)}$ of approximation (5).

\section{Lemma 3}

$$
\begin{gathered}
\gamma_{n-1}^{(\alpha)}=\frac{26-\alpha}{24 \Gamma(-\alpha) n^{1+\alpha}}+O\left(\frac{1}{n^{2+\alpha}}\right) \\
\gamma_{n}^{(\alpha)}=-\frac{1}{\Gamma(1-\alpha) n^{\alpha}}+\frac{13 \alpha+10}{24 \Gamma(-\alpha) n^{1+\alpha}}+O\left(\frac{1}{n^{2+\alpha}}\right) .
\end{gathered}
$$

Proof

$$
\begin{aligned}
\gamma_{n}^{(\alpha)}=W_{n}^{1}-W_{n}^{0} & =-\frac{1}{\Gamma(1-\alpha) n^{\alpha}}+\left(\frac{\alpha+1}{2}+\frac{\alpha-2}{24}\right) \frac{1}{\Gamma(-\alpha) n^{1+\alpha}}+O\left(\frac{1}{n^{2+\alpha}}\right), \\
\gamma_{n}^{(\alpha)} & =-\frac{1}{\Gamma(1-\alpha) n^{\alpha}}+\frac{13 \alpha+10}{24 \Gamma(-\alpha) n^{1+\alpha}}+O\left(\frac{1}{n^{2+\alpha}}\right) .
\end{aligned}
$$

From (8) with $n:=n-1$

$$
w_{n-1}^{(\alpha)}=(-1)^{n}\left(\begin{array}{c}
\alpha \\
n-1
\end{array}\right)=\frac{1}{\Gamma(-\alpha)(n-1)^{1+\alpha}}+O\left(\frac{1}{(n-1)^{2+\alpha}}\right),
$$




$$
w_{n-1}^{(\alpha)}=\frac{1}{\Gamma(-\alpha) n^{1+\alpha}}+O\left(\frac{1}{n^{2+\alpha}}\right) .
$$

Therefore

$$
\begin{gathered}
\gamma_{n-1}^{(\alpha)}=w_{n-1}^{(\alpha)}-W_{n}^{1}=\frac{1}{\Gamma(-\alpha) n^{1+\alpha}}-\frac{\alpha-2}{24 \Gamma(-\alpha) n^{1+\alpha}}+O\left(\frac{1}{n^{2+\alpha}}\right), \\
\gamma_{n-1}^{(\alpha)}=\frac{26-\alpha}{24 \Gamma(-\alpha) n^{1+\alpha}}+O\left(\frac{1}{n^{2+\alpha}}\right) .
\end{gathered}
$$

Now we construct a second-order approximation of the Caputo derivative at the point $x_{n}-\alpha h / 2$ by replacing the weights $\gamma_{k}^{(\alpha)}$ of approximation (30) which have an index $k>\lceil N / 5\rceil$ with the first terms of their expansion formulas (8), (35) and (36):

$$
\frac{1}{h^{\alpha}} \sum_{k=0}^{n} \bar{\gamma}_{k}^{(\alpha)} y_{n-k}=y_{n-\alpha / 2}^{(\alpha)}+O\left(h^{2}\right) .
$$

When $n \leq\lceil N / 5\rceil$ the weights of approximation (37) are equal to the weights of approximation (30): $\bar{\gamma}_{k}^{(\alpha)}=w_{k}^{(\alpha)}=(-1)^{k}\left(\begin{array}{l}\alpha \\ k\end{array}\right)$ for $0 \leq k \leq n-2$ and

$$
\bar{\gamma}_{n-1}^{(\alpha)}=\frac{n-2 \alpha}{1+\alpha-n} w_{n-2}^{(\alpha-2)}+\frac{(n-\alpha / 2)^{1-\alpha}}{\Gamma(2-\alpha)}, \bar{\gamma}_{n}^{(\alpha)}=w_{n-2}^{(\alpha-2)}-\frac{(n-\alpha / 2)^{1-\alpha}}{\Gamma(2-\alpha)} .
$$

When $n>\lceil N / 5\rceil$ the weights $\bar{\gamma}_{k}^{(\alpha)}$ of approximation (37) satisfy

$$
\begin{gathered}
\bar{\gamma}_{k}^{(\alpha)}= \begin{cases}w_{k}^{(\alpha)}=(-1)^{k}\left(\begin{array}{l}
\alpha \\
k
\end{array}\right), & 0 \leq k \leq\lceil N / 5\rceil, \\
\frac{1}{\Gamma(-\alpha) k^{1+\alpha}}-\frac{\alpha}{2 \Gamma(-1-\alpha) k^{2+\alpha}},\lceil N / 5\rceil<k=n-2,\end{cases} \\
\bar{\gamma}_{n-1}^{(\alpha)}=\frac{26-\alpha}{24 \Gamma(-\alpha) n^{1+\alpha}}, \bar{\gamma}_{n}^{(\alpha)}=-\frac{1}{24 \Gamma(1-\alpha) n^{\alpha}}+\frac{13 \alpha+10}{24 \Gamma(-\alpha) n^{1+\alpha}} .
\end{gathered}
$$

\section{Numerical results}

The fractional relaxation equation is a two-term ordinary fractional differential equation, where $0<\alpha<1$ :

$$
y^{(\alpha)}(x)+L y(x)=f(x), \quad y(0)=y_{0} .
$$

From (2) with $n=1$ we obtain an approximation of order $2-\alpha$ for the Caputo derivative at the point at $x=h$ :

$$
y^{(\alpha)}(h)=\frac{y(h)-y(0)}{\Gamma(2-\alpha) h^{\alpha}}+O\left(h^{2-\alpha}\right) .
$$


By approximating the Caputo derivative in equation (38) with (39) we obtain

$$
\begin{gathered}
\frac{y(h)-y(0)}{\Gamma(2-\alpha) h^{\alpha}}+L y(h)=f(h)+O\left(h^{2-\alpha}\right), \\
\widetilde{y}_{1}=\frac{y(0)+\Gamma(2-\alpha) h^{\alpha} f(h)}{1+\Gamma(2-\alpha) L h^{\alpha}}=y(h)+O\left(h^{2}\right) .
\end{gathered}
$$

The number $\widetilde{y}_{1}$ is a second order approximation for the value of the solution of equation (38) at $x=h$. Suppose that

$$
\frac{1}{h^{\alpha}} \sum_{k=0}^{n} \lambda_{k}^{(\alpha)} y_{n-k}=y_{n}^{(\alpha)}+O\left(h^{\beta}\right)
$$

is an approximation of the Caputo derivative of order $\beta$, where $\beta \leq 2$. In (Dimitrov 2016) we obtain the numerical solution of equation (38) on the interval $[0,1]$ which uses approximation $(*)$ for the Caputo derivative.

$$
u_{n}=\frac{1}{\lambda_{0}^{(\alpha)}+L h^{\alpha}}\left(h^{\alpha} f_{n}-\sum_{k=1}^{n-1} \lambda_{k}^{(\alpha)} u_{n-k}\right), u_{0}=y_{0}, u_{1}=\widetilde{y}_{1}
$$

Suppose that

$$
\frac{1}{h^{\alpha}} \sum_{k=0}^{n} \lambda_{k}^{(\alpha)} y_{n-k}=y_{n-\alpha / 2}^{(\alpha)}+O\left(h^{2}\right)
$$

is a second-order shifted approximation of the Caputo derivative with a shift parameter $-\alpha h / 2$. In (Dimitrov 2014) we show that the numerical solution of equation (38) which uses approximation $(* *)$ of the Caputo derivative is computed with $u_{0}=y_{0}, u_{1}=\widetilde{y}_{1}$ and

$$
u_{n}=\frac{1}{\lambda_{0}^{(\alpha)}+L\left(1-\frac{\alpha}{2}\right) h^{\alpha}}\left(h^{\alpha} f_{n}-\frac{\alpha L h^{\alpha}}{2} u_{n-1}-\sum_{k=1}^{n-1} \lambda_{k}^{(\alpha)} u_{n-k}\right) .
$$

\section{Example 1:}

$$
y^{(\alpha)}(x)+y(x)=2 x^{2+\alpha}+\Gamma(3+\alpha) x^{2}, y(0)=0,
$$

Equation (40) has the solution $y(x)=2 x^{2+\alpha}$. The solution of equation (40) satisfies the condition $y(0)=y^{\prime}(0)=0$. The numerical results for the error and the order of second-order numerical solution NS2(9) of equation (40) are presented in Table 1. The numerical results for the error and the order of numerical solution NS2(16) of order $2-\alpha$ and the second-order numerical solution NS2(17) of equation (40) are presented in Table 2 and Table 3. The exponential function has a Caputo derivative $D^{\alpha} e^{x}=x^{1-\alpha} E_{1,2-\alpha}(x)$, where $E_{a, b}(x)$ is the Mittag-Leffler function $E_{a, b}=\sum_{n=0}^{\infty} \frac{x^{n}}{\Gamma(a n+b)}$. The sine and cosine functions have Caputo derivatives

$$
D^{\alpha} \sin x=x^{1-\alpha} E_{2,2-\alpha}\left(-x^{2}\right), D^{\alpha} \cos x=-x^{2-\alpha} E_{2,3-\alpha}\left(-x^{2}\right) .
$$


Table 1 Maximum error and order of numerical solution NS2(9) of equation 40 when $\alpha=0.2, \alpha=0.5$ and $\alpha=0.9$.

\begin{tabular}{|l|cc|cc|cc|}
\hline \multirow{2}{*}{\multicolumn{1}{|c|}{$\boldsymbol{h}$}} & \multicolumn{2}{|c|}{$\boldsymbol{\alpha}=\mathbf{0 . 2}$} & \multicolumn{2}{c|}{$\boldsymbol{\alpha}=\mathbf{0 . 5}$} & \multicolumn{2}{c|}{$\boldsymbol{\alpha}=\mathbf{0 . 9}$} \\
\cline { 2 - 7 } & Error & Order & Error & Order & Error & Order \\
\hline 0.00625 & $6.9 \times 10^{-6}$ & 1.9868 & 0.00002911 & 1.9742 & 0.00004546 & 1.9774 \\
0.003125 & $1.7 \times 10^{-6}$ & 1.9934 & $7.3 \times 10^{-6}$ & 1.9871 & 0.00001145 & 1.9889 \\
0.0015625 & $4.4 \times 10^{-7}$ & 1.9967 & $1.8 \times 10^{-6}$ & 1.9936 & $2.8 \times 10^{-6}$ & 1.9945 \\
0.00078125 & $1.1 \times 10^{-7}$ & 1.9983 & $4.6 \times 10^{-7}$ & 1.9968 & $7.2 \times 10^{-7}$ & 1.9973 \\
\hline
\end{tabular}

Table 2 Maximum error and order of numerical solution NS2(16) of equation (40) when $\alpha=0.2, \alpha=0.5$ and $\alpha=0.9$.

\begin{tabular}{|l|cc|cc|cc|}
\hline \multirow{2}{*}{\multicolumn{1}{|c|}{$\boldsymbol{h}$}} & \multicolumn{2}{|c|}{$\boldsymbol{\alpha}=\mathbf{0 . 2}$} & \multicolumn{2}{c|}{$\boldsymbol{\alpha}=\mathbf{0 . 5}$} & \multicolumn{2}{c|}{$\boldsymbol{\alpha}=\mathbf{0 . 9}$} \\
\cline { 2 - 7 } & Error & Order & Error & Order & Error & Order \\
\hline 0.00625 & $7.9 \times 10^{-6}$ & 1.9092 & 0.00007985 & 1.5675 & 0.00044332 & 1.1490 \\
0.003125 & $2.1 \times 10^{-6}$ & 1.9031 & 0.00002728 & 1.5494 & 0.00020323 & 1.1252 \\
0.0015625 & $5.7 \times 10^{-7}$ & 1.8965 & $9.4 \times 10^{-6}$ & 1.5358 & 0.00009398 & 1.1127 \\
0.00078125 & $1.5 \times 10^{-7}$ & 1.8897 & $3.3 \times 10^{-6}$ & 1.5257 & 0.00004365 & 1.1063 \\
\hline
\end{tabular}

Table 3 Maximum error and order of numerical solution NS2(17) of equation (40) when $\alpha=0.2, \alpha=0.5$ and $\alpha=0.9$.

\begin{tabular}{|l|cc|cc|cc|}
\hline \multirow{2}{*}{\multicolumn{1}{|c|}{$\boldsymbol{h}$}} & \multicolumn{2}{|c|}{$\boldsymbol{\alpha}=\mathbf{0 . 2}$} & \multicolumn{2}{c|}{$\boldsymbol{\alpha}=\mathbf{0 . 5}$} & \multicolumn{2}{c|}{$\boldsymbol{\alpha}=\mathbf{0 . 9}$} \\
\cline { 2 - 7 } & Error & Order & Error & Order & Error & Order \\
\hline 0.00625 & $4.3 \times 10^{-6}$ & 1.9993 & $9.3 \times 10^{-6}$ & 2.0239 & 0.00001743 & 2.1067 \\
0.003125 & $1.1 \times 10^{-6}$ & 1.9998 & $2.3 \times 10^{-6}$ & 2.0188 & $4.0 \times 10^{-6}$ & 2.1181 \\
0.0015625 & $2.7 \times 10^{-7}$ & 2.0000 & $5.7 \times 10^{-7}$ & 2.0143 & $9.2 \times 10^{-7}$ & 2.1256 \\
0.00078125 & $6.7 \times 10^{-8}$ & 2.0000 & $1.4 \times 10^{-7}$ & 2.0106 & $2.1 \times 10^{-7}$ & 2.1313 \\
\hline
\end{tabular}

The digamma function is the logarithmic derivative of the gamma function

$$
\psi(x)=\frac{d}{d x} \ln \Gamma(x)=\frac{\Gamma^{\prime}(x)}{\Gamma(x)} .
$$

The digamma function satisfies:

$$
\psi(1)=-\gamma, \quad \psi(n)=H_{n-1}-\gamma, \quad \psi(x+1)=\psi(x)+\frac{1}{x},
$$

where $H_{n}=\sum_{k=1}^{n} \frac{1}{k}$ is the $n$-th harmonic number and $\gamma=0.5772 \ldots$ is the Euler-Mascheroni constant. The Caputo derivative of order $\alpha$ of the function $y(x)=x^{3} \ln x$ satisfies

$$
\begin{gathered}
D^{\alpha} x^{3} \ln x=\frac{\Gamma(4) x^{3-\alpha}}{\Gamma(4-\alpha)}(\ln x+\psi(4)-\psi(4-\alpha)), \\
D^{\alpha} x^{3} \ln x=\frac{x^{3-\alpha}}{\Gamma(4-\alpha)}(11+6 \ln x-6 \gamma-6 \psi(4-\alpha)) .
\end{gathered}
$$

\section{Example 2:}

$$
\begin{aligned}
& y^{(\alpha)}(x)+y(x)=\sin x+\cos x+x^{3} \ln x+x^{1-\alpha} E_{2,2-\alpha}\left(-x^{2}\right)- \\
& x^{2-\alpha} E_{2,3-\alpha}\left(-x^{2}\right)+\frac{x^{3-\alpha}}{\Gamma(4-\alpha)}(11+6 \ln x-6 \gamma-6 \psi(4-\alpha)), y(0)=1 .
\end{aligned}
$$


Equation (48) has an exact solution $y(x)=\sin x+\cos x+x^{3} \ln x$. The solution satisfies $y(0)=y^{\prime}(0)=1$. The numerical results for the error and the order of numerical solution NS1(22) of order $2-\alpha$ and second-order numerical solutions NS2(25), NS2(30), NS2(37) of equation (48) are presented in Table 4, Table 5, Table 6 and Table 7.

Table 4 Maximum error and order of numerical solution NS1(22) of equation (41) when $\alpha=0.2, \alpha=0.5$ and $\alpha=0.9$.

\begin{tabular}{|l|cc|cc|cc|}
\hline \multirow{2}{*}{\multicolumn{1}{|c|}{$\boldsymbol{h}$}} & \multicolumn{2}{|c|}{$\boldsymbol{\alpha}=\mathbf{0 . 2}$} & \multicolumn{2}{c|}{$\boldsymbol{\alpha}=\mathbf{0 . 5}$} & \multicolumn{2}{c|}{$\boldsymbol{\alpha}=\mathbf{0 . 9}$} \\
\cline { 2 - 7 } & Error & Order & Error & Order & Error & Order \\
\hline 0.00625 & 0.00001516 & 1.8429 & 0.00009894 & 1.5343 & 0.00115363 & 1.0966 \\
0.003125 & $4.2 \times 10^{-6}$ & 1.8429 & 0.00003438 & 1.5251 & 0.00053881 & 1.0983 \\
0.0015625 & $1.2 \times 10^{-6}$ & 1.8395 & 0.00001201 & 1.5174 & 0.00025150 & 1.0992 \\
0.00078125 & $3.3 \times 10^{-7}$ & 1.8332 & $4.4 \times 10^{-6}$ & 1.5029 & 0.00011736 & 1.0996 \\
\hline
\end{tabular}

Table 5 Maximum error and order of numerical solution NS1(25) of equation (41) when $\alpha=0.2, \alpha=0.5$ and $\alpha=0.9$.

\begin{tabular}{|l|cc|cc|cc|}
\hline \multirow{2}{*}{\multicolumn{1}{|c|}{$\boldsymbol{h}$}} & \multicolumn{2}{|c|}{$\boldsymbol{\alpha}=\mathbf{0 . 2}$} & \multicolumn{2}{c|}{$\boldsymbol{\alpha}=\mathbf{0 . 5}$} & \multicolumn{2}{c|}{$\boldsymbol{\alpha}=\mathbf{0 . 9}$} \\
\cline { 2 - 7 } & Error & Order & Error & Order & Error & Order \\
\hline 0.00625 & 0.00001771 & 1.9405 & 0.00002919 & 1.8905 & 0.00002368 & 2.1213 \\
0.003125 & $4.5 \times 10^{-6}$ & 1.9686 & $7.6 \times 10^{-6}$ & 1.9346 & $5.6 \times 10^{-6}$ & 2.0908 \\
0.0015625 & $1.1 \times 10^{-6}$ & 1.9833 & $1.9 \times 10^{-6}$ & 1.9596 & $1.3 \times 10^{-6}$ & 2.0588 \\
0.00078125 & $2.9 \times 10^{-7}$ & 1.9910 & $5.0 \times 10^{-7}$ & 1.9742 & $3.3 \times 10^{-7}$ & 2.0368 \\
\hline
\end{tabular}

Table 6 Maximum error and order of numerical solution NS2(5) of equation 41 when $\alpha=0.2, \alpha=0.5$ and $\alpha=0.9$.

\begin{tabular}{|l|cc|cc|cc|}
\hline \multirow{2}{*}{\multicolumn{1}{|c|}{$\boldsymbol{h}$}} & \multicolumn{2}{|c|}{$\boldsymbol{\alpha}=\mathbf{0 . 2}$} & \multicolumn{2}{c|}{$\boldsymbol{\alpha}=\mathbf{0 . 5}$} & \multicolumn{2}{c|}{$\boldsymbol{\alpha}=\mathbf{0 . 9}$} \\
\cline { 2 - 7 } & Error & Order & Error & Order & Error & Order \\
\hline 0.00625 & $3.3 \times 10^{-6}$ & 1.9951 & $6.7 \times 10^{-6}$ & 2.0589 & 0.00001766 & 2.0905 \\
0.003125 & $8.2 \times 10^{-7}$ & 1.9976 & $1.6 \times 10^{-6}$ & 2.0339 & $4.2 \times 10^{-6}$ & 2.0591 \\
0.0015625 & $2.1 \times 10^{-7}$ & 1.9988 & $4.1 \times 10^{-7}$ & 2.0173 & $1.0 \times 10^{-6}$ & 2.0363 \\
0.00078125 & $5.1 \times 10^{-8}$ & 1.9994 & $1.0 \times 10^{-7}$ & 2.0075 & $2.5 \times 10^{-7}$ & 2.0214 \\
\hline
\end{tabular}

Table 7 Maximum error and order of numerical solution NS2(37) of equation 41] when $\alpha=0.2, \alpha=0.5$ and $\alpha=0.9$.

\begin{tabular}{|l|cc|cc|cc|}
\hline \multirow{2}{*}{\multicolumn{1}{|c|}{$\boldsymbol{h}$}} & \multicolumn{2}{|c|}{$\boldsymbol{\alpha}=\mathbf{0 . 2}$} & \multicolumn{2}{c|}{$\boldsymbol{\alpha = \mathbf { 0 . 5 }}$} & \multicolumn{2}{c|}{$\boldsymbol{\alpha}=\mathbf{0 . 9}$} \\
\cline { 2 - 7 } & Error & Order & Error & Order & Error & Order \\
\hline 0.00625 & 0.00009038 & 1.9180 & 0.00019044 & 1.9240 & 0.00013348 & 1.9553 \\
0.003125 & 0.00002322 & 1.9603 & 0.00004891 & 1.9611 & 0.00003389 & 1.9776 \\
0.0015625 & $5.9 \times 10^{-6}$ & 1.9787 & 0.00001239 & 1.9801 & $8.5 \times 10^{-6}$ & 1.9889 \\
0.00078125 & $1.5 \times 10^{-6}$ & 1.9893 & $3.1 \times 10^{-6}$ & 1.9901 & $2.1 \times 10^{-6}$ & 1.9946 \\
\hline
\end{tabular}


The analytical solutions of the system of ordinary fractional differential equations

$$
\left|\begin{array}{l}
y^{(\alpha)}(x)+A y(x)+B z(x)=f(x) \\
z^{(\alpha)}(x)+C y(x)+D z(x)=g(x)
\end{array}\right|
$$

is in studied in (Ertürk and Momani 2008; Diethlm et al. 2017). Now we obtain the numerical solution NS3(*) of (42) which uses approximation (*) of the Caputo derivative. By approximating the Caputo derivative of $y(x)$ and $z(x)$ at the point $x_{n}=n h$ in both equations of (42) we obtain

$$
\begin{aligned}
& \frac{1}{h^{\alpha}} \sum_{k=0}^{n} \lambda_{k}^{(\alpha)} y_{n-k}+A y_{n}+B z_{n}=f_{n}+O\left(h^{\beta}\right), \\
& \frac{1}{h^{\alpha}} \sum_{k=0}^{n} \lambda_{k}^{(\alpha)} z_{n-k}+C y_{n}+D z_{n}=g_{n}+O\left(h^{\beta}\right) .
\end{aligned}
$$

Let $\left\{u_{n}\right\}_{n=0}^{N}$ and $\left\{v_{n}\right\}_{n=0}^{N}$ be the numerical solutions of (42) on the net $\left\{x_{n}\right\}_{n=0}^{N}$, where $u_{n}$ is an approximation of $y_{n}$ and $v_{4}$ is an approximation for the value of $z_{n}$. From (43) and (44) the numbers $u_{n}$ and $v_{n}$ satisfy the following system of linear equations:

$$
\mid \begin{aligned}
& \left(\lambda_{0}^{(\alpha)}+A h^{\alpha}\right) u_{n}+B h^{\alpha} v_{n}=S_{n} \\
& C h^{\alpha} u_{n}+\left(\lambda_{0}^{(\alpha)}+D h^{\alpha}\right) v_{n}=Q_{n}
\end{aligned}
$$

where

$$
S_{n}=h^{\alpha} f_{n}-\sum_{k=1}^{n} \lambda_{k}^{(\alpha)} u_{n-k}, \quad Q_{n}=h^{\alpha} g_{n}-\sum_{k=1}^{n} \lambda_{k}^{(\alpha)} v_{n-k}
$$

Denote $\widetilde{D}=\left(\lambda_{0}^{(\alpha)}+A h^{\alpha}\right)\left(\lambda_{0}^{(\alpha)}+D h^{\alpha}\right)-B C h^{2 \alpha}$. The numbers $u_{n}$ and $v_{n}$ are the solutions of 45

$$
\begin{gathered}
u_{n}=\frac{1}{\widetilde{D}}\left(\left(\lambda_{0}^{(\alpha)}+D h^{\alpha}\right) S_{n}-B h^{\alpha} Q_{n}\right), \\
v_{n}=\frac{1}{\widetilde{D}}\left(-C h^{\alpha} S_{n}+\left(\lambda_{0}^{(\alpha)}+A h^{\alpha}\right) Q_{n}\right) .
\end{gathered}
$$

From (46) and (47) with $n=1$ and approximation (39) for $y_{1}^{(\alpha)}$ and $z_{1}^{(\alpha)}$ we obtain

$$
\begin{aligned}
& \widetilde{u}_{1}=\frac{1}{\widetilde{D}}\left(\left(\sigma_{0}^{(\alpha)}+D h^{\alpha}\right)\left(h^{\alpha} f(h)+\sigma_{0}^{(\alpha)} y_{0}\right)-B h^{\alpha}\left(h^{\alpha} g(h)+\sigma_{0}^{(\alpha)} z_{0}\right)\right) \\
& \widetilde{v}_{1}=\frac{1}{\widetilde{D}}\left(\left(\sigma_{0}^{(\alpha)}+A h^{\alpha}\right)\left(h^{\alpha} g(h)+\sigma_{0}^{(\alpha)} z_{0}\right)-C h^{a}\left(h^{\alpha} f(h)+\sigma_{0}^{(\alpha)} y_{0}\right)\right)
\end{aligned}
$$

where $\sigma_{0}^{(\alpha)}=1 / \Gamma(2-\alpha)$. The numbers $\widetilde{u}_{1}$ and $\widetilde{v}_{1}$ are second-order approximations for the values of the solutions $y_{1}$ and $z_{1}$ of (42). Numerical solution 
$\mathrm{NS} 3\left(^{*}\right)$ has initial conditions $u_{0}=y_{0}, u_{1}=\widetilde{u}_{1}, v_{0}=z_{0}, v_{1}=\widetilde{v}_{1}$ and and the numbers $u_{n}$ and $v_{n}$, for $2 \leq n \leq N$ are computed with (46) and (47).

\section{Example 3:}

$$
\mid \begin{aligned}
& y^{(\alpha)}(x)+y(x)+2 z(x)=2 e^{x}+e^{2 x}+2^{\alpha} x^{1-\alpha} E_{1,2-\alpha}(2 x) \\
& z^{(\alpha)}(x)+3 y(x)+4 z(x)=4 e^{x}+3 e^{2 x}+x^{1-\alpha} E_{1,2-\alpha}(x) .
\end{aligned}
$$

The solution of the sytem of equations (48) is $y(x)=e^{2 x}, z(x)=e^{x}$. The numerical results for the error and the order of numerical solution NS3(22) of order $2-\alpha$ and the second-order numerical solution NS3(25) of the solution $y(x)=e^{2 x}$ of the system of fractional differential equations (48) are presented in Table 8 and Table 9 .

Now we obtain the numerical solution NS4(**) of (42) which uses the second-order shifted approximation $(* *)$ of the Caputo derivative. By approximating $y_{n-\alpha / 2}^{(\alpha)}$ and $z_{n-\alpha / 2}^{(\alpha)}$ with $(* *)$ we obtain

$$
\begin{aligned}
& \frac{1}{h^{\alpha}} \sum_{k=0}^{n} \lambda_{k}^{(\alpha)} y_{n-k}+A y_{n-\alpha / 2}+B z_{n-\alpha / 2}=f_{n-\alpha / 2}+O\left(h^{2}\right), \\
& \frac{1}{h^{\alpha}} \sum_{k=0}^{n} \lambda_{k}^{(\alpha)} z_{n-k}+C y_{n-\alpha / 2}+D z_{n-\alpha / 2}=g_{n-\alpha / 2}+O\left(h^{2}\right) .
\end{aligned}
$$

From (49) and (50) and the second order approximations

$y_{n-\alpha / 2}=\frac{\alpha}{2} y_{n-1}+\left(1-\frac{\alpha}{2}\right) y_{n}+O\left(h^{2}\right), z_{n-\alpha / 2}=\frac{\alpha}{2} z_{n-1}+\left(1-\frac{\alpha}{2}\right) z_{n}+O\left(h^{2}\right)$,

we obtain the system of equations for $u_{n}$ and $v_{n}$

$$
\mid \begin{aligned}
& \left(\lambda_{0}^{(\alpha)}+A h^{\alpha}\left(1-\frac{\alpha}{2}\right)\right) u_{n}+B h^{\alpha}\left(1-\frac{\alpha}{2}\right) v_{n}=S_{n} \\
& C h^{\alpha}\left(1-\frac{\alpha}{2}\right) u_{n}+\left(\lambda_{0}^{(\alpha)}+D h^{\alpha}\left(1-\frac{\alpha}{2}\right)\right) v_{n}=Q_{n}
\end{aligned}
$$

where

$$
\begin{aligned}
& S_{n}=h^{\alpha}\left(f_{n-\alpha / 2}-\frac{\alpha}{2}\left(A u_{n-1}+B v_{n-1}\right)\right)-\sum_{k=1}^{n} \lambda_{k}^{(\alpha)} u_{n-k}, \\
& Q_{n}=h^{\alpha}\left(g_{n-\alpha / 2}-\frac{\alpha}{2}\left(C u_{n-1}+D v_{n-1}\right)\right)-\sum_{k=1}^{n} \lambda_{k}^{(\alpha)} v_{n-k} .
\end{aligned}
$$

Denote

$$
\widehat{D}=\left(\lambda_{0}^{(\alpha)}+A h^{\alpha}\left(1-\frac{\alpha}{2}\right)\right)\left(\lambda_{0}^{(\alpha)}+D h^{\alpha}\left(1-\frac{\alpha}{2}\right)\right)-B C\left(1-\frac{\alpha}{2}\right)^{2} h^{2 \alpha} .
$$


Numerical solution NS4(**) of the system of fractional differential equations (42) has initial conditions $u_{0}=y_{0}, u_{1}=\widetilde{u}_{1}, v_{0}=z_{0}, v_{1}=\widetilde{v}_{1}$. The numbers $u_{n}$ and $v_{n}$ are computed with:

$$
\begin{gathered}
u_{n}=\frac{1}{\widehat{D}}\left(\left(\lambda_{0}^{(\alpha)}+D h^{\alpha}\left(1-\frac{\alpha}{2}\right)\right) S_{n}-B h^{\alpha}\left(1-\frac{\alpha}{2}\right) Q_{n}\right), \\
v_{n}=\frac{1}{\widehat{D}}\left(-C h^{\alpha}\left(1-\frac{\alpha}{2}\right) S_{n}+\left(\lambda_{0}^{(\alpha)}+A h^{\alpha}\left(1-\frac{\alpha}{2}\right)\right) Q_{n}\right) .
\end{gathered}
$$

The numerical results for the error and the order of second-order numerical solutions NS4(30) and NS4(37) of the solution $y(x)$ of the system of fractional differential equations (48) are presented in Table 10 and Table 11.

Table 8 Maximum error and order of numerical solution NS3(22) of the solution $y(x)$ of (48) when $\alpha=0.2, \alpha=0.5$ and $\alpha=0.9$.

\begin{tabular}{|l|cc|cc|cc|}
\hline \multirow{2}{*}{\multicolumn{1}{|c|}{$\boldsymbol{h}$}} & \multicolumn{2}{|c|}{$\boldsymbol{\alpha}=\mathbf{0 . 2}$} & \multicolumn{2}{c|}{$\boldsymbol{\alpha}=\mathbf{0 . 5}$} & \multicolumn{2}{c|}{$\boldsymbol{\alpha}=\mathbf{0 . 9}$} \\
\cline { 2 - 7 } & Error & Order & Error & Order & Error & Order \\
\hline 0.00625 & 0.00023740 & 1.6637 & 0.00213254 & 1.4523 & 0.02108710 & 1.0981 \\
0.003125 & 0.00007385 & 1.6846 & 0.00077199 & 1.4659 & 0.00984709 & 1.0986 \\
0.0015625 & 0.00002267 & 1.7038 & 0.00027752 & 1.4761 & 0.00459682 & 1.0991 \\
0.00078125 & $6.9 \times 10^{-6}$ & 1.7201 & 0.00009927 & 1.4831 & 0.00214536 & 1.0994 \\
\hline
\end{tabular}

Table 9 Maximum error and order of numerical solution NS3(25) of the solution $y(x)$ of (48) when $\alpha=0.2, \alpha=0.5$ and $\alpha=0.9$.

\begin{tabular}{|l|cc|cc|cc|}
\hline \multirow{2}{*}{\multicolumn{1}{|c|}{$\boldsymbol{h}$}} & \multicolumn{2}{|c|}{$\boldsymbol{\alpha}=\mathbf{0 . 2}$} & \multicolumn{2}{c|}{$\boldsymbol{\alpha}=\mathbf{0 . 5}$} & \multicolumn{2}{c|}{$\boldsymbol{\alpha}=\mathbf{0 . 9}$} \\
\cline { 2 - 7 } & Error & Order & Error & Order & Error & Order \\
\hline 0.00625 & 0.00003134 & 1.9964 & 0.00005672 & 2.0110 & 0.00008675 & 2.0370 \\
0.003125 & $7.8 \times 10^{-6}$ & 1.9983 & 0.00001409 & 2.0086 & 0.00002119 & 2.0355 \\
0.0015625 & $1.9 \times 10^{-6}$ & 1.9993 & $3.5 \times 10^{-6}$ & 2.0065 & $5.2 \times 10^{-6}$ & 2.0311 \\
0.00078125 & $4.9 \times 10^{-6}$ & 1.9997 & $8.7 \times 10^{-7}$ & 2.0048 & $1.2 \times 10^{-6}$ & 2.0292 \\
\hline
\end{tabular}

Table 10 Maximum error and order of numerical solution NS4(5) of the solution $y(x)$ of (48) when $\alpha=0.2, \alpha=0.5$ and $\alpha=0.9$.

\begin{tabular}{|l|cc|cc|cc|}
\hline \multirow{2}{*}{\multicolumn{1}{|c|}{$\boldsymbol{h}$}} & \multicolumn{2}{|c|}{$\boldsymbol{\alpha}=\mathbf{0 . 2}$} & \multicolumn{2}{c|}{$\boldsymbol{\alpha}=\mathbf{0 . 5}$} & \multicolumn{2}{c|}{$\boldsymbol{\alpha}=\mathbf{0 . 9}$} \\
\cline { 2 - 7 } & Error & Order & Error & Order & Error & Order \\
\hline 0.00625 & $2.7 \times 10^{-6}$ & 1.9959 & $6.1 \times 10^{-6}$ & 1.9668 & 0.00001588 & 1.9937 \\
0.003125 & $6.9 \times 10^{-7}$ & 1.9979 & $1.5 \times 10^{-6}$ & 1.9747 & $3.9 \times 10^{-6}$ & 1.9963 \\
0.0015625 & $1.7 \times 10^{-7}$ & 1.9989 & $3.9 \times 10^{-7}$ & 1.9812 & $9.9 \times 10^{-7}$ & 1.9979 \\
0.00078125 & $4.3 \times 10^{-8}$ & 1.9994 & $9.9 \times 10^{-8}$ & 1.9863 & $2.5 \times 10^{-7}$ & 1.9988 \\
\hline
\end{tabular}


Table 11 Maximum error and order of numerical solution NS4(37) of the solution $y(x)$ of (48) when $\alpha=0.2, \alpha=0.5$ and $\alpha=0.9$.

\begin{tabular}{|l|cc|cc|cc|}
\hline \multirow{2}{*}{\multicolumn{1}{|c|}{$\boldsymbol{h}$}} & \multicolumn{2}{|c|}{$\boldsymbol{\alpha}=\mathbf{0 . 2}$} & \multicolumn{2}{c|}{$\boldsymbol{\alpha}=\mathbf{0 . 5}$} & \multicolumn{2}{c|}{$\boldsymbol{\alpha}=\mathbf{0 . 9}$} \\
\cline { 2 - 7 } & Error & Order & Error & Order & Error & Order \\
\hline 0.00625 & 0.00009952 & 2.0137 & 0.00020851 & 2.0089 & 0.00015518 & 1.9852 \\
0.003125 & 0.00002479 & 2.0055 & 0.00005198 & 2.0042 & 0.00003900 & 1.9928 \\
0.0015625 & $6.2 \times 10^{-6}$ & 2.0018 & 0.00001298 & 2.0019 & $9.8 \times 10^{-6}$ & 1.9960 \\
0.00078125 & $1.5 \times 10^{-6}$ & 2.0009 & $3.2 \times 10^{-6}$ & 2.0009 & $2.4 \times 10^{-6}$ & 1.9979 \\
\hline
\end{tabular}

\section{Conclusions}

In the present paper we showed that the properties of the approximations of the Caputo derivative are preserved when the weights with an index greater than $\lceil N / p\rceil$ are replaced by the first terms of their asymptotic expansions, where $p$ is a positive number. In section 4 we obtained an approximation (5) of the Caputo derivative by modifying the last two weights of the Grünwald-Letnikov approximation. Approximation (5) is a second-order shifted approximation for the Caputo derivative for all functions $y \in C^{2}[0, x]$. In future work we are going to apply the methods and the approximations of the Caputo derivative discussed in the paper for numerical solution of fractional differential equations with singular and non-singular solutions.

\section{References}

1. Alikhanov AA (2015) A new difference scheme for the time fractional diffusion equation. Journal of Computational Physics 280: 424-438

2. Bhrawy AH, Taha TM, Alzahrani EO, Baleanu D, Alzahrani AA (2015) New operational matrices for solving fractional differential equations on the half-line. PLoS ONE. 10(5): e0126620

3. Chen M, Deng W (2014) Fourth Order Accurate Scheme for the Space Fractional Diffusion Equations. SIAM Journal on Numerical Analysis 52(3): 1418-1438

4. Diethelm K, Siegmund S, Tuan HT (2017) Asymptotic behavior of solutions of linear multi-order fractional differential systems. Fractional Calculus and Applied Analysis 20(5): 1165-1195.

5. Dimitrov Y (2014) Numerical approximations for fractional differential equations. Journal of Fractional Calculus and Applications 5(3S): 1-45

6. Dimitrov Y (2016) Second-order approximation for the Caputo derivative. Journal of Fractional Calculus and Applications 7(2): 175-195

7. Dimitrov Y, Miryanov R, Todorov V (2017) Quadrature formulas and Taylor series of secant and tangent. Economics and computer science 4: $23-40$

8. Dimitrov Y (2018) Approximations for the Caputo derivative (I). Journal of Fractional Calculus and Applications 9(1): 35-63 
9. Ding H, Li C (2016) High-order algorithms for Riesz derivative and their applications (III). Fractional Calculus and Applied Analisys 19(1): 19-55

10. Ding H, Li C (2017) High-order numerical algorithms for Riesz derivatives via constructing new generating functions. Journal of Scientific Computing 71(2): 759-784

11. El-Borai MM, El-Sayed WG, Jawad AM (2015) Adomian decomposition method for solving fractional differential equations. International Research Journal of Engineering and Technology 2(6): 295-306

12. Elezović N (2005) Asymptotic expansions of gamma and related functions, binomial coefficients, inequalities and means. Journal of Mathematical Inequalities 9(4): 1001-1054

13. Ertürk VS, Momani S (2008) Solving systems of fractional differential equations using differential transform method. Journal of Computational and Applied Mathematics 215: 142-151

14. Ezz-Eldien SS, Hafez RM, Bhrawy AH, Baleanu D, El-Kalaawy AA (2017) New numerical approach for fractional variational problems using shifted Legendre orthonormal polynomials. Journal of Optimization Theory and Applications 174 (1): 295-320

15. Gao GH, Sun ZZ, Zhang HW (2014) A new fractional numerical differentiation formula to approximate the Caputo fractional derivative and its applications. Journal of Computational Physicss 259: 33-50

16. Gao GH, Sun HW, Sun ZZ (2015) Stability and convergence of finite difference schemes for a class of time-fractional sub-diffusion equations based on certain superconvergence. Journal of Computational Physics 280: 510-528

17. Jin B, Lazarov R, Zhou Z (2016) An analysis of the L1 scheme for the subdiffusion equation with nonsmooth data. IMA Journal of Numerical Analysis 36(1): 197-221

18. Li C, Chen A, Ye J (2011) Numerical approaches to fractional calculus and fractional ordinary differential equation. Journal of Computational Physics 230(9): $3352-3368$.

19. Lin Y, Xu C (2007) Finite difference/spectral approximations for the timefractional diffusion equation. Journal of Computational Physics 225: 1533 1552

20. Lubich C (1986) Discretized fractional calculus. SIAM Journal on Mathematical Analysis 17(3): 704-719

21. Magin RL (2004) Fractional calculus in bioengineering. Critical Reviews in Biomedical Engineering 32(1): 1-104

22. Monje CA, Chen YQ, Vinagre BM, Xue D, Feliu-Batlle V (2010) Fractional-order Systems and Controls: Fundamentals and Applications. Springer Science \& Business Media.

23. Pedas A, Tamme E (2014) Numerical solution of nonlinear fractional differential equations by spline collocation methods. Journal of Computational and Applied Mathematics 255: 216-230

24. Podlubny I (1999) Fractional Differential Equations. Academic Press, San Diego 
25. Ren L, Wang YM (2017) A fourth-order extrapolated compact difference method for time-fractional convection-reaction-diffusion equations with spatially variable coefficients. Applied Mathematics and Computation 312: $1-22$

26. Tadjeran C, Meerschaert MM, Scheffer HP (2006) A second-order accurate numerical approximation for the fractional diffusion equation. Journal of Computational Physics 213: 205-213

27. Tian W, Zhou H, Deng W (2015) A class of second order difference approximations for solving space fractional diffusion equations. Mathematics of Computation 84: 1703-1727

28. Tricomi F, Erdélyi A (1951) The asymptotic expansion of a ratio of gamma functions. Pacific Journal of Mathematics. 1(1): 133-142

29. Vong S, Wang Z (2014) High order difference schemes for a time-fractional differential equation with Neumann boundary conditions. East Asian Journal on Applied Mathematics 4(3): 222-241

30. Wang S, Xu M (2007) Generalized fractional Schrödinger equation with space-time fractional derivatives. Journal of Mathematical Physics 48: 043502

31. Yan Y, Pal K, Ford NJ (2014) Higher order numerical methods for solving fractional differential equations. BIT Numerical Mathematics 54: 555-584

32. Zayernouri M, Karniadakis GE (2014) Exponentially accurate spectral and spectral element methods for fractional ODEs. Journal of Computational Physics 257: 460-480

33. Zhang H, Liu F, Turner I, Chen S (2016) The numerical simulation of the tempered fractional Black-Scholes equation for European double barrier option. Applied Mathematical Modelling, Applied Mathematical Modelling, 40(1112):5819-5834

34. Zheng M, Liu F, Liu Q, Burrage K, Simpson MJ (2017) Numerical solution of the time fractional reactiondiffusion equation with a moving boundary. Journal of Computational Physics 338: 493-510

35. Zhuang P, Liu F (2006) Implicit difference approximation for the time fractional diffusion equation. Journal of Applied Mathematics and Computing 22(3): 87-99 